\title{
Carbon Nanotube Nanofluidics
}

\author{
Jong Won Choi, Maria A. Alexandrova and Hyung Gyu Park* \\ Swiss Federal Institute of Technology Zurich (ETH Zürich), Zürich, \\ Switzerland
}

\section{Introduction}

Carbon nanotubes (CNT), with diameters in the nanometer range and atomically smooth surfaces, offer a unique system for studying molecular transport and nanofluidics. Although the idea that water can occupy such confined hydrophobic channels is somewhat counterintuitive, experimental evidence has confirmed that water can indeed occupy these channels (Naguib, Ye et al. 2004; Kolesnikov, Loong et al. 2006). Water transport through molecularscale hydrophobic channels is also important because of the similarity of this system to transmembrane protein pores such as aquaporins (Agre, Borgnia et al. 2001; Agre 2004; Agre 2006). In recent years, numerous simulations (Hummer, Rasaiah et al. 2001; Kalra 2003) of water transport through single-walled carbon nanotubes (SWNT) have suggested not only that water occupies these channels, but also that fast molecular transport takes place, far in excess of what continuum hydrodynamic theories would predict if applied on this length scale. Molecular dynamics (MD) simulations attribute this enhancement to the molecular-level smoothness of the nanotube interior surface and to molecular ordering phenomena that may occur on confined length scales in the 1- to 2-nm range (Hummer, Rasaiah et al. 2001; Skoulidas, Ackerman et al. 2002; Kalra 2003). For similar reasons, simulations of gas transport through SWNTs (Lai, Bonilla et al. 2003) predict flux enhancements of several orders of magnitude relative to other similarly sized nanoporous materials. Membrane-based gas separations, such as those using zeolites (Hinds, Chopra et al. 2004), provide precise separation and size exclusion, although often at the expense of throughput or flux. It may be possible to use SWNT to create a membrane that offers both high selectivity and high flux.

To investigate molecular transport on this length scale, we need to fabricate a carbon nanotube membrane that has a pore size of the order of $1 \mathrm{~nm}$. Researchers have recently fabricated multi-walled carbon nanotube (MWNT) membranes with larger pore diameters (6 to $7 \mathrm{~nm}$ ) by encapsulation of vertically aligned arrays of MWNTs (Hinds, Chopra et al. 2004; Holt, Noy et al. 2004) and by templated growth within nanochannel alumina (Li, Papadopoulos et al. 1999). Enhanced water transport through these larger MWNTs has recently been reported (MajumderMainak, ChopraNitin et al. 2005). Quantifying transport through an individual tube in a MWNT membrane is difficult, however, because MWNTs are prone to blockages, in particular by "bamboo" structures and catalyst particles that can migrate to and obstruct the nanotube interior (Cui, Zhou et al. 2000; Maruyama, Einarsson et al. 2005; Wang, Gupta et al. 2005). The consequence of such blockages is a marked reduction

*parkh@ethz.ch 
of the active membrane pore density. In contrast, there are few, if any, reports of "bamboo" structure formation or catalyst migration in SWNTs or double-walled carbon nanotubes (DWNTs). However, it has been difficult to produce vertically aligned carbon nanotubes of this size uniformly and at large scale (Kalra 2003; Hata, Futaba et al. 2004). The major challenges also lie in finding a conformal deposition process to fill the gaps in this nanotube array, as well as in designing a selective etching process to open up the nanotube channels without producing voids in the membrane. These challenges in nanomanufacturing are one of the major reasons for the imbalance between the number of reports from computational and experimental studies, offering great research opportunities in the area of experimental Carbon Nanotube Nanofluidics.

This book chapter is intended to provide an intermediate level overview of Carbon Nanotube Nanofluidics to the beginners and scientists who are interested in this emerging research field. For that purpose, we write discussions of Carbon Nanotube Nanofluidics with respect to transporting entities. In section 2, we discuss water transport under the CNT nanoconfinement in view of the unique transport phenomena including spontaneous water filling, fast water transport and mechanisms behind it. In section 3, we discuss the various aspects of gas transport in CNT. We present fundamental findings of Carbon Nanotube Nanofluidics for the gas transport, followed by a perspective of gas separation using CNT membranes. In section 4, we introduce recent achievements made by theoretical and experimental studies focusing on the behavior of ions in CNT including transport of ions under the CNT nanoconfinement, and ion exclusion and selectivity in association with CNT membrane technology. Finally, we will conclude several aspects of Carbon Nanotube Nanofluidics in section 5 .

\section{Water transport}

\subsection{Introduction and overview}

Nanoscale confinement carbon nanotube $(\mathrm{CNT})$ provides can lead to transport phenomena unique at this length scale featured by spontaneous water filling and fast water transport. It is the graphitic curvature of the internal space of CNT that effectively smoothes the potential energy landscape to cause minimal friction to water flows. The last decade saw growing attention on the flow enhancement of pressure-driven water through CNT membranes that exceeds the continuum theory prediction by three to four orders of magnitude. This phenomenon renders CNT an attractive building block for various nanofluidic applications such as molecular sensors, nanopipettes, hydrogen storage, membrane filtration, drug delivery, energy conversion and dissipation, in which the hydrophobicity, atomic-level smoothness and nanoscale pore size interplay to result in considerable improvement of energy efficiency.

Conduits of CNT consist of rolled sheets of graphene with $\mathrm{sp}^{2}$-hybridized carbon atoms that are not generally known to be wetted by water. In view of continuum models, it seems incredible that polar liquids, especially water, may fill in such narrow hydrophobic CNT conduits. However, at nanometer scales where surmises of those continuum models no longer hold, water molecules can behave somewhat differently from macroscopic predictions, mostly characterized by ordered configuration of the hydrogen bond network. Hummer et al. (Hummer, Rasaiah et al. 2001) used equilibrium molecular dynamics (MD) simulation about 
an $8 \AA$ wide $(6,6)$ CNT in a water bath to argue that water molecules do not only enter the narrow hydrophobic channel spontaneously, but can also move very fast through it. They supported the argument of spontaneous filling by comparison of water binding energy distributions between bulk and nanoconfinement states. Unlike Gaussian distribution of bulk water binding energies centered around a value corresponding to ca. four hydrogen bonds, the binding energy distribution under the nanoconfinement showed degeneracy featured by population inversion at lower binding energies. In other words, lower binding energies are less populated by water under the nanoconfinement of CNT, leading to tight hydrogen bonding network of a single file and a decrease in chemical potential, a thermodynamic explanation of the spontaneous filling of CNT conduits with water molecules.

To support the next part of the argument (rapid water conduction), Hummer et al. (Hummer, Rasaiah et al. 2001) counted water molecules passing through CNT on a computational domain. Even though average flow rate estimate was zero because their simulation was equilibrium molecular dynamics, most of the constituent microstates involved burst-like motions of the single-file water molecules as fast as $17 \mathrm{~ns}^{-1}$. Another simulation on the water transport through a membrane that consists of open-ended $(6,6)$ CNTs was performed under an osmotic pressure gradient by Kalra et al. (Kalra 2003). They obtained the motion of about $5 \mathrm{~ns}^{-1}$ and found that single-file water molecules are governed by microscopic fluctuations and nearly independent of the tube length due to very low friction coefficient. These theoretical predictions were verified with experimental studies of Holt, Park et al. (Holt 2006), in which the water flow was measured through microfabricated CNT membranes with pores less than $2 \mathrm{~nm}$. The measured water flow rates exceeded the prediction of a continuum hydrodynamics model by more than three orders of magnitude. Majumder et al. (Majumder, Chopra et al. 2005) also made an investigation of the water transport through CNT membrane with large pores of around 7 $\mathrm{nm}$. In this experiment, the measured flow rates were four to five orders of magnitude larger than a continuum hydrodynamics model. Joseph and Aluru (Joseph and Aluru 2008) simulated the pressure-driven flow in a $(16,16)$ CNT to reveal that the enhanced flow rate over Hagen-Poiseuille formalism is primarily caused by a velocity "jump" in a depletion region between the nearest water layer and the carbon wall. They concluded that a slightly modified water-carbon interaction potential to make the CNT wall surface more hydrophilic could greatly reduce the enhancement in the water transport because it would lower down the number of hydrogen atoms pointing toward the carbon wall in the depletion layer. Nanoscale roughness in the tube walls caused no significant flow enhancement. Additional phenomena and effects of nanoconfinement in CNTs on the water structure, density profile, hydrogen bonds, and some suggestions about the fast water transport are illustrated in detail in the following sections. This chapter begins with explaining the classical theory of fluid mechanics briefly, limit of the application of the theories, and hydrophobic Interaction between the water molecules and CNT. Subsequently, using several literatures for water transport under nanoconfinement of CNT, we will summarize water structure in CNTs, water density profile in CNTs, and hydrogen bonds of water inside CNTs. In the next, we will enlist some theoretical descriptions about the main principles and mechanisms of infiltration and transport of water in CNT. Finally, we will discuss nanofluidic applications and some perspectives the ultrafast water transport poses potential of. 


\subsection{Continuum limit of water}

Continuum model of water hypothesizes that the spatial and temporal derivatives of all the macroscopic dependent variables such as density, velocity, stress, and heat flux exist in a continuous and differentiable manner. Navier-Stokes equation derived from the force balance has been representatively used for explaining the dynamic behavior of fluids such as diffusion, conduction, and momentum transport in the macro scale, where the physical properties such as density and velocity profiles can vary appreciably over the molecular length and relaxation time scales regardless of the nature of the molecular structure and configuration of a fluid under consideration. However, in nanoscale conduits of CNT, much larger flow rates are observed for the fluid transport that cannot be explained by the continuum theory. Derived from these phenomena, one may raise several questions: How does the Navier-Stokes equation break down at the nanoscales? Can we still use the continuum-based theories to predict the behavior such as diffusion, heat transfer, or momentum transport? Why would the water inside CNT have anything specific for the transport? Indeed, there have been many efforts to define the boundary between bulk water and confined water transport. A liquid fluid particle can be heuristically defined as a volume containing more than 10,000 molecules because we need at least 10,000 molecules for obtaining less than $1 \%$ statistical fluctuation in measuring physical properties. Thus, one may reasonably consider the liquid particle of 10,000 molecules as a continuum limit. Since a sphere containing an aggregate of 10,000 equidistant molecules has an equivalent diameter of about 25 molecules and the size of a water molecule is estimated as ca. $0.3 \mathrm{~nm}$, the diameter of the 10,000 water molecules will be calculated as about $7.5 \mathrm{~nm}$. A tube with the diameter of much larger than $7.5 \mathrm{~nm}$ can allow the fundamental hypothesis of hydrodynamics. Hence, it would be sensible to set a threshold for the continuum treatment of liquid as around $7.5 \mathrm{~nm}$.

In the non-continuum regime, there exists another threshold associated with variation in fluid properties. Raviv and Klein (Raviv 2002) asserted that water loses its bulk viscosity below 1-2 nm, with a drastic change for stronger confinements. They presented the wettability of confining surface plays a dominant role for the viscosity below 1-2 nm. This threshold was emphasized again by other researchers (Lei and Leng 2010; Thomas, Iutzi et al. 2010). These two threshold values could impart guideline values in the analysis of nanoscale molecular transport phenomena.

\subsection{Slip length theory}

A no slip boundary condition is typically used in continuum fluid dynamics. It constrains a fluid closest to a solid boundary to obtain the same tangential velocity as the solid. When the tangential velocity of fluid differs from that of solid, we usually say the surface imposes a slip boundary condition as depicted in Fig. 2.1. One may wonder whether the slippery motion of water in a nanoscale hydrophobic tube of CNT can be well described using continuum equations. Nevertheless, the slip boundary condition helps us describe non continuum behavior of water transport inside CNT in the framework of continuum dynamics. For example, slip length, $\mathrm{L}_{\mathrm{s}}$, may serve as a good indicator for the molecular interaction between water molecules and CNT via provision of information about the degree of departure the transport innately has from the hydrodynamic Hagen-Poiseuille flow. Also, the slip length indicator can compare with results of MD simulations often used for the exact prediction of water flow under the CNT nanoconfinement. 


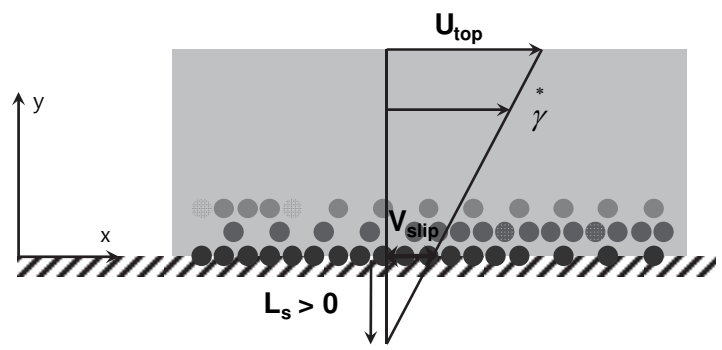

Fig. 2.1. Hydrodynamic slip flow profile characterized by slip length, $L_{\mathrm{s}}$.

Unfortunately, there exists no definite criterion or nondimensional number that categorizes liquid flow regimes. In this situation, the Knudsen number used for distinguishing the gas flow regimes may serve as an indirect gauge that could tell us about the liquid flow regime. It is defined as the ratio of mean free path to characteristic length. Well accepted criteria for gas flows are given as follows (Steckelmacher 1986).

$$
\begin{array}{cr}
\mathrm{Kn} & <0.001 \\
0.001<\mathrm{Kn}<0.1 & \text { (Continuum flow) } \\
0.1<\mathrm{Kn}<10 & \text { (Slip flow) } \\
10<\mathrm{Kn} & \text { (Free molecular flow) }
\end{array}
$$

In liquid, molecules are rather densely aggregated and they interact one another within the length scale of the molecule's own size. If one would define a pseudo mean free path of liquid water as the molecular size, the Knudsen number for the tube having diameter of 1 $\mathrm{nm}$ is calculated to be 0.15 , placing the transport between slip flow and transition flow regimes. Hence, continuum hydrodynamics may not be allowed to define the velocity profile in narrow $\mathrm{CNT}$. Slip length, $\mathrm{L}_{\mathrm{s}}$, is convenient to explain the hydrodynamic boundary condition at the interface of fluid and wall, which is defined according to the Navier boundary condition in equation (2.1).

$$
\left.L_{s} \frac{\partial v_{t}}{\partial n}\right|_{\text {wall }}=v_{t, \text { wall }}-v_{\text {wall }}
$$

where $n$ and $t$ denote normal and tangential directions of the wall, $v_{\mathrm{t}}$ is the velocity of a fluid tangential to the wall, and $v_{\text {wall }}$ is the velocity of the wall. $v_{t, \text { wall }}-v_{\text {wall }}$ is denoted as a slip velocity. In some sense, the slip length is related to the surface friction at the fluid-wall interface. Larger slip lengths can be related with lower liquid-solid friction. For example, the slip length at solid surfaces depends on the wettability of the surface: easily wetted surface may exert larger friction. Therefore, very large slip lengths of water flows obtained in CNT signify a considerable enhancement in water flow rates (Lee, Choi et al. 2008). As the size of the nanotube decreases, the dynamics at its surface plays an increasingly important role (Willmott and Tallon 2007). For investigating the relationship between the slip length and the interfacial properties, it is useful to understand the slip length in connection of liquid-solid interface friction. The friction force is linearly proportional to slip velocity as in 
equation (2.2). By definition, the slip length is related with a friction coefficient: equation (2.3).

$$
\begin{gathered}
F_{\text {friction }}=-A_{L} f\left(v_{\mathrm{t}, \text { wall }}-v_{\text {wall }}\right), \\
L_{s}=\mu / f,
\end{gathered}
$$

where $f$ is the friction coefficient, $A_{L}$ is the lateral area, and $\mu$ is the bulk viscosity.

An approximation of the Green-Kubo relation can give an expression for the friction coefficient $f$ : equation (2.4) (Sendner, Horinek et al. 2009).

$$
f \approx \frac{1}{A_{L} k_{B} T}\left\langle F_{\text {friction }}^{2}\right\rangle_{\text {equilibrium }} \times \tau,
$$

where $\tau$ is the relaxation time, $\left\langle F^{2}\right\rangle_{\text {equilibrium }}$ is the rms friction force, and $k_{\mathrm{B}} T$ is the reference thermal energy. The relaxation time is written by $\tau \approx \sigma^{2} / D$ where $\sigma$ is the scale of molecular diameter and $D$ is the fluid diffusion coefficient. From equations (2.3) and (2.4), we can estimate the function of slip length as

$$
L_{s} \approx \frac{A_{L} k_{B} T \mu D}{\sigma^{2}\left\langle F_{\text {friction }}^{2}\right\rangle_{\text {equilibrium }}},
$$

According to equation (2.5), a large degree of flow slippage implying a weak liquid-solid interaction is expected to occur at high temperatures or on very smooth surfaces (low friction force). The solutions for the velocity and the corresponding volume flow rate in the flow direction, $z$, with respect to the distance from the center, $r$, have a parabolic profile given by:

$$
\begin{gathered}
U_{\mathrm{s}}=-\frac{d p}{d z} \frac{R^{2}}{4 \mu}\left(1-\frac{r^{2}}{R^{2}}+\frac{2 L_{s}}{R}\right), \\
Q_{\text {Hagen-Poiseuille }}=-\frac{d p}{d z} \frac{\pi R^{4}}{8 \mu}, \\
Q_{\mathrm{s}}=Q_{\text {Hagen-Poiseuille }}\left(1+\frac{4 L_{\mathrm{s}}}{R}\right),
\end{gathered}
$$

where $\mu, p$, and $R$ represent viscosity, pressure, and tube radius, respectively.

There are two primary reasons to investigate a continuum theory of a molecular scale slip behavior in this section. On the one hand, it helps to understand the behavior of a flow in nanoscale tube, thereby predicting what kinds of factors or physical mechanisms are responsible for the flow enhancement. On the other hand, the slip length theory can serve as a reference to explain large water flow enhancements, compared with no-slip Hagen-Poiseuille formalism, observed by several experimental studies using CNT membranes. 
Researchers used the slip length theory to discuss their experimental results with CNT membranes (Majumder, Chopra et al. 2005; Holt 2006; Whitby, Cagnon et al. 2008). By use of a MWNT membrane with a pore diameter of $7 \mathrm{~nm}$, Hinds and colleagues reported largely enhanced flow rate per pore, beyond any theoretical expectation. Bakajin and colleagues also measured water flow enhancement through a DWNT membrane based on nanocomposite out of vertically aligned CNTs having a pore size of 1.3-2 nm and silicon nitride. They all quoted corresponding slip lengths of 53,728 nm (enhancement factor of 61,404 (Majumder, Chopra et al. 2005)) and 140 to 1,400 nm (enhancement factor of 560-8,400 (Holt 2006)). Later experiments performed through carbon nanopipes with diameters of 44 nm suggested a slip length of 32-38 nm (enhancement factor of 22-24 (Whitby, Cagnon et al. 2008)). In the absence of enough experimental data to judge, it is still a question which results are more acceptable yet or how the enhancement factor is related with the tube size, creating a need of more experimental verifications out of various degrees of CNT nanoconfinements for obtaining reasonable enhancement values and relationship between the enhancement value and nanotube geometry. Although a challenge in manufacturing remains, additional experimental efforts will provide the answers to the questions such as when the continuum theory breaks down and what kinds of theory would explain the fluidics inside CNT better.

\subsection{Water structure and hydrogen bonds}

Although the ultrafast water transport in the CNT could, in part, be explained by the slip length model, it is indeed well reported that the water flow rate depends not on bulk properties such as density and viscosity but rather on the interaction between water molecules and carbon atoms in the CNT wall. Accordingly, one can model the relation between the surface friction and structure of water molecules inside CNT. In this section, instead of slip flow theory, results of MD simulations, especially on the water configuration, are introduced and compared since it is a stronger and effective way to investigate many fundamental nanofluidic characteristics. While water molecules inside CNT conduct in a single file configuration when the tube becomes narrow down to a few water molecular size (i.e. sub $1 \mathrm{~nm}$ ), the structure of water in wider CNTs seems to take a layered configuration especially near the internal walls.

A hydrogen bond found in water is an attractive interaction between a hydrogen atom of one water molecule and an electronegative atom, oxygen of another water molecule. It is stronger than a van der Waals interaction, but weaker than covalent or ionic bonds. Actually, lots of physical properties of water depend on the coherence and the number of the hydrogen bonds. The hydrogen bond is useful in explaining water configuration or properties inside CNT, since water has a dipole and an electrostatic interaction via hydrogen bonding. It is well known that each water molecule can have approximately 3.6 of hydrogen bonded neighbor molecules in bulk water. Many results of MD simulations support that the average hydrogen bond between water molecules decreases in confined tube and the confined water molecules lose two hydrogen bonds on average from the bulk configuration, since there is a lack of space to retain the bulk state in such a narrow nanotube, and therefore this value reaches approximately 1.6 bonds in narrow CNTs, where only a single file of water is allowed as shown in Fig. 2.2 (Hummer, Rasaiah et al. 2001; Eschermann, Li et al. 2006; Banerjee, Murad et al. 2007; Hanasaki, Nakamura et al. 2008). 


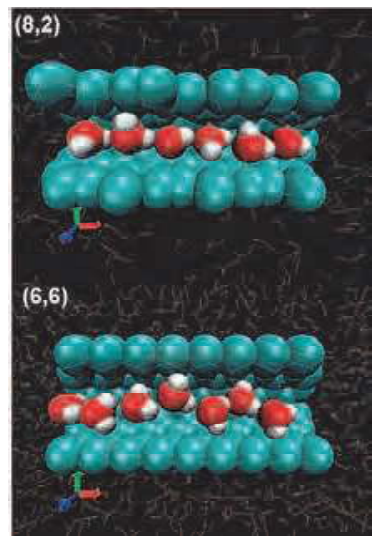

Fig. 2.2. Single-file configuration of water molecules in $(8,2)$ and $(6,6)$ carbon nanotubes (Alexiadis and Kassinos 2008).

Byl et al. (Byl, Liu et al. 2006) assessed that water molecules are highly oriented and aligned hand in hand along the axis of a single-file chain, thereby allowing water molecules to rotate freely around the aligned hydrogen bonds. Study on the single file was introduced by Hummer and colleagues (Hummer, Rasaiah et al. 2001) using water molecules in $8 \AA$-wide $(6,6)$ SWNT at the pressure of 1 bar. They found counter intuitively that water molecules may configure themselves in a single file, allowing only two hydrogen bonds per molecule, and occupy the CNT spontaneously during the entire simulation period of $66 \mathrm{~ns}$. This occupancy, nevertheless, can degrade into fluctuation between filled- and empty states when the water-tocarbon interaction potential is slightly rendered more hydrophobic. They explained that it is not because of the average water binding energy in CNT (enthalpic contribution) but because of the local excess chemical potential (enthalpo-entropic contribution) that determines the water occupancy of narrow CNTs. Kolesnikov and his group (Kolesnikov, Loong et al. 2006) provided evidence that water can indeed fill inside narrow $(10,10)$ CNT and pose a configuration of a single file inside an octagonal water nanotube. Mashl et al. (Mashl, Joseph et al. 2003) simulated the similar situation to predict a hexagonal water inside $(9,9)$ SWNT at 1 bar, but they did not find any similar structure among $(7,7),(8,8)$, or $(10,10)$ CNTs. However, MD simulation by Byl et al. (Byl, Liu et al. 2006) did not find any distinguishable water structure inside CNTs from $(7,7)$ to $(10,10)$, while they found a single file formation in $(5,5)$ SWNTs by using different potential parameter. Even with a large number of efforts to determine the water layer structure in CNTs with various diameters, the results are still different from one another. This discrepancy can be explained by diverse water models and water-to-carbon interaction potentials. For example, a lower interaction potential value is likely to allow confined water to form n-gonal structures easily, while a higher value tends to keep water molecules disordered. However, it is certain that all the simulations show that water confined in CNTs shows characteristics that considerably differ from those of bulk water, regardless of selected water models. Recent study on the water structure on temperature and pressure was carried out by Koga et al. (Koga, Gao et al. 2001). They found that water took an n-gonal configuration between 500 and 5,000 bars, and lifetime of defect (break and reunion) was longer for wider CNTs. They concluded that the number of concentric water nanotubes could be determined by the nanotube chirality (i.e. with $n=4,5$, 
and 6 for $(14,14),(15,15)$, and $(16,16)$ CNTs, respectively.) Likewise, Lin et al. (Lin, Shiomi et al. 2009) showed the radial density profile and water structure using different density values inside SWNTs of $(6,6),(8,8),(10,10)$, and $(14,14)$. They presented the water could fill into the tube for the density of $1.4 \mathrm{~g} / \mathrm{cm}^{3}$ among the water densities of $1.0,1.2$, and $1.4 \mathrm{~g} / \mathrm{cm}^{3}$ at least except for $(6,6)$ SWNT, which was partially filled spontaneously for all the given densities. The maximum water peaks are slightly increased as the diameters increase due to the flexibility of the SWNTs. The water configurations for different diameters of CNT are shown in Fig. 2.3. As the tube diameters increase from $(6,6)$ to $(14,14)$, water configuration in CNT evolved from a single-file chain to a single wall water nanotube, to a single file in a single wall water nanotube, and finally to a single file in a multi-walled water nanotube. Using these simulation results, Alexiadis and Kassinos (Alexiadis and Kassinos 2008) calculated diameter dependence of an average number of the hydrogen bonds, determined based on the criteria of oxygen-tooxygen distance of $3.3 \AA$, oxygen-to-hydrogen distance of $2.4 \AA$, and bonding angle between $\mathrm{O}-\mathrm{O}$ direction and $\mathrm{H}-\mathrm{O}$ direction of less than $30^{\circ}$ : Fig. 2.4. The diagram of $\mathrm{H}$-bond number vs. CNT diameter allowed them to identify distinctive water structures such as "single-file water", "water layers", and "bulk mode." However, more recent experimental work carried by Kyakuno et al. (Kyakuno, Matsuda et al. 2010) did not support this finding. They constructed a phase diagram of water inside SWNT as shown in Fig. 2.5, based on the XRD (green squares), NMR (red circles), and two MD simulation sources from Shiomi et al. (Shiomi, Kimura et al. 2007) (black squares) and Takaiwa et al. (Takaiwa, Koga et al. 2007) (blue triangles). The approximate diameter near the boundary between liquid and ice is around $1.5 \mathrm{~nm}$, in which they proposed that there exist double or triple shell geometries. In narrower CNTs, $1.17<d<$ $1.45 \mathrm{~nm}$, a new water configuration named ice nanotubes has the transition freezing points similar with ice. As inferable from these results, it would be meaningful to establish the phase of water for $1.17<d<1.45 \mathrm{~nm}$ and at near room temperature.

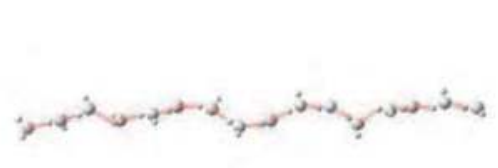

(a)

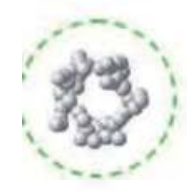

(c)

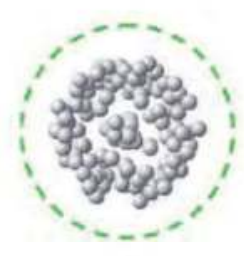

(d)

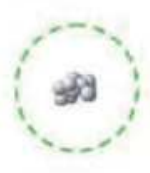

(b)

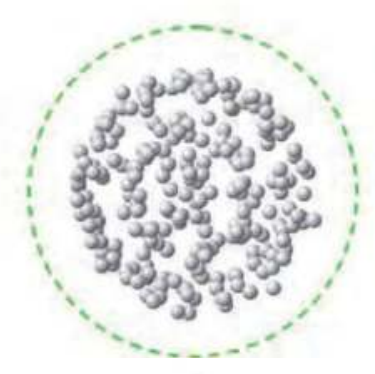

(e)

Fig. 2.3. Water inside SWNTs with different diameters. (a) side view of water inside $(6,6)$ SWNT (b) cross section in $(6,6)$ SWNT, (c) $(8,8)$ SWNT, (d) $(10,10)$ SWNT, (e) $(14,14)$ SWNT. Only oxygen atoms are shown for clarity (Lin, Shiomi et al. 2009). 


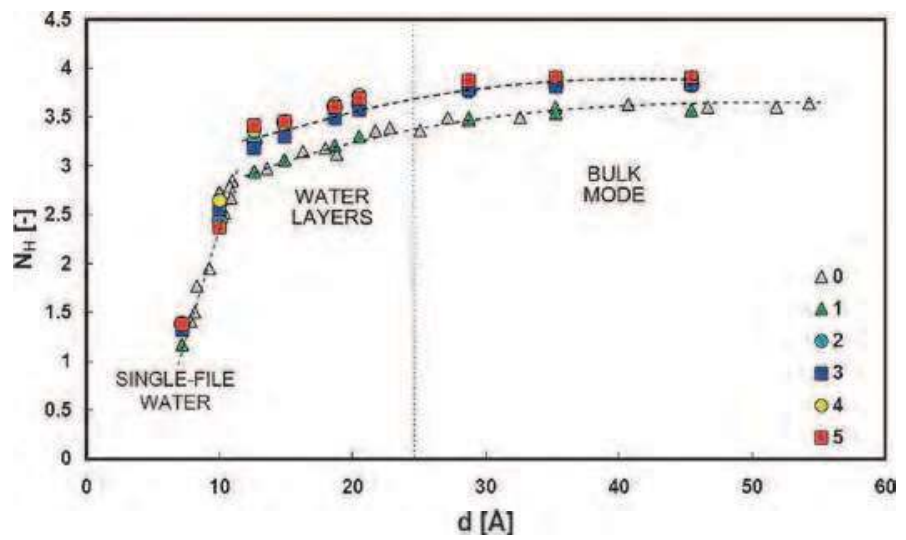

Fig. 2.4. Hydrogen bonding (calculated with $R_{\mathrm{OO}}=3.3 \AA, R_{\mathrm{HO}}=2.4 \AA$, and $\varphi=30^{\circ}$ ) versus diameter. The data are divided into six different groups: (0) TIP3P water model/rigid CNT; (1) TIP3P water model/flexible CNT; (2) SPC/E water model/rigid CNT; (3) flexible TIP3P water model/rigid CNT; (4) flexible SPC water model/rigid CNT; and (5) flexible SPC water model/flexible CNT (Alexiadis and Kassinos 2008).

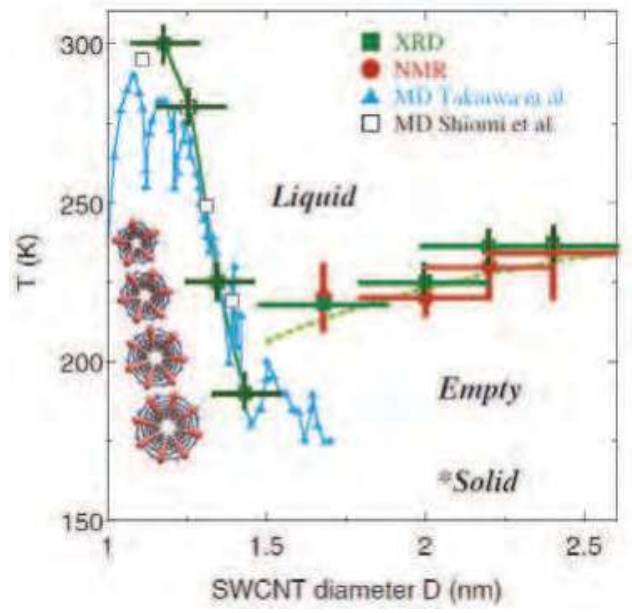

Fig. 2.5. Global temperature-diameter (T-D) phase diagram of water inside SWNT (Kyakuno, Matsuda et al. 2010).

\subsection{Radial and axial water density distribution}

Probing of water density distribution is probably a promising way to understand the confinement effect because it can give intuition about a possible water layer existing in CNT. Pozhar (Pozhar 2000) pointed out that the dynamics of liquid flows such as a radial density distribution is not influenced by fluid but only by intermolecular potentials such as van der Waals interaction potential through investigation of simple Lennard-Jones fluids. Hanasaki and Nakatami (Hanasaki and Nakatani 2006) carried out an MD simulation on radial 
density distribution of water confined under SWNTs of different sizes from $(6,6)$ to $(20,20)$ to confirm that water configuration depends primarily on the tube diameter. Fig. 2.6 shows relative local radial density profiles (local density/bulk density) of water with respect to various diameters of CNTs (Alexiadis and Kassinos 2008). In a very narrow tube like $(6,6)$ SWNT, only one water peak could be observed because there is no more space between a single-file water wire and the graphitic wall except a depletion region. As CNT diameter increases, it is sensible to expect the second peak and a maximum water peak sifted toward the carbon wall, which means that the center line is less favored energetically. The number of water layers, $\mathrm{n}$, with a given tube radius also depends on both $\sigma_{\mathrm{OO}}$ and $\sigma_{\mathrm{CO}}$, and can be calculated by the following equation (Wang, Zhu et al. 2004).

$$
n=1+\frac{r-\sigma_{\mathrm{CO}}}{\sigma_{\mathrm{OO}}}
$$

Wang et al. (Wang, Zhu et al. 2004) presented that the water in CNT tends gradually to behave as bulk water when the molecules are placed beyond three or four water layers away from the wall because the confinement effect on density weakens so that the radial density distribution may converge to that of water on a graphite slab. While the radial density distribution represents water layering across the diameter of CNT, it is the axial density distribution that explains how much the water molecule is affected by hexagonal cells of carbon. Therefore, if the radial and axial density distributions are obtained, the overall water density in CNTs can be calculated by integrating these two profiles in the nanotube. Thomas and McGaughey (Thomas and McGaughey 2008) investigated the confinement effects on the radial density profile inside and outside tubes for SWNT diameters of 1.1, 2.8, 6.9, and 10.4 $\mathrm{nm}$ to suggest a criterion for the CNT radius to distinguish confined water from unconfined water. For example, both inside and outside the $10.4 \mathrm{~nm}-\mathrm{CNT}$, the water density distribution looks exactly the same as that on a flat graphene sheet. The unconfined water density outside the CNT showed the first local maximum at $r=R_{c}+0.3 \mathrm{~nm}$, the local minimum at $r=R_{c}+0.5 \mathrm{~nm}$, and the second local maximum at $r=R_{c}+0.7 \mathrm{~nm}$, where $R_{c}$ is the radius measured between the centerline and the center of carbon atoms of CNT. As the CNT diameter decreases, however, the density profiles took different shapes between inside and outside of CNT because surface curvature of narrow tubes could effectively reduce the water density by pulling water molecules away from the surface. As a result, the maximum density was located at $r=R_{c}-0.3 \mathrm{~nm}$ and $r=R_{c}-1.3 \mathrm{~nm}$ for tubes with diameters of 1.1 and $2.8 \mathrm{~nm}$, respectively. Zhou et al. (Zhou and $\mathrm{Lu} 2007$ ) investigated the axial density distributions for $(6,6),(8,8)$ and $(9,9)$ SWNTs, which looks like periodic corrugation along the axis regardless of the radius. The distance between two water peaks was about $2.5 \AA$, almost same as the distance between two carbon atoms of a hexagonal cell. Alexiadis and Kassinos (Alexiadis and Kassinos 2008) studied the water density profiles using several water models and flexible/rigid nanotubes to suggest that the water molecules should exist in the form of a "single-file" or "layered" structure inside CNT with diameter below $24.8 \AA$ A. They also examined how water density distribution can vary with respect to the tube diameter. If the nanotube diameter decreases from $(8,2)$ to $(6,6)$, the density increases since the volume of the nanotube decreases although the number of molecules per nanotube length does not change. On the other hand, as the tube diameter increased from about $1 \mathrm{~nm}$ to $24.8 \mathrm{~nm}$, the water density decreased because CNT could allow more than one water layers. As a consequence, water molecules located at the centerline of CNT can behave as bulk water if 
the diameter further increases. The following correlation was derived from Fig. 2.6, which can be used to approximate the water density in nanotubes with different diameters.

$$
\begin{gathered}
\frac{\rho}{\rho_{0}}=1-\frac{\rho_{1}^{*}}{\left(d / d_{1}^{*}+1\right)^{2}}, \text { with } d_{1}^{*}=20.6 \dot{\mathrm{A}}, \\
\rho_{1}^{*}=1.4 \mathrm{~g} / \mathrm{cm}^{3} \text { for } d<24.8 \dot{\mathrm{A}} \\
\frac{\rho}{\rho_{0}}=1-\frac{\rho_{2}^{*}}{\left(d / d_{2}^{*}+1\right)^{2}}, \text { with } d_{2}^{*}=55.0 \dot{\mathrm{A}}, \\
\rho_{2}^{*}=0.6 \mathrm{~g} / \mathrm{cm}^{3} \text { for } d \geq 24.8 \dot{\mathrm{A}}
\end{gathered}
$$
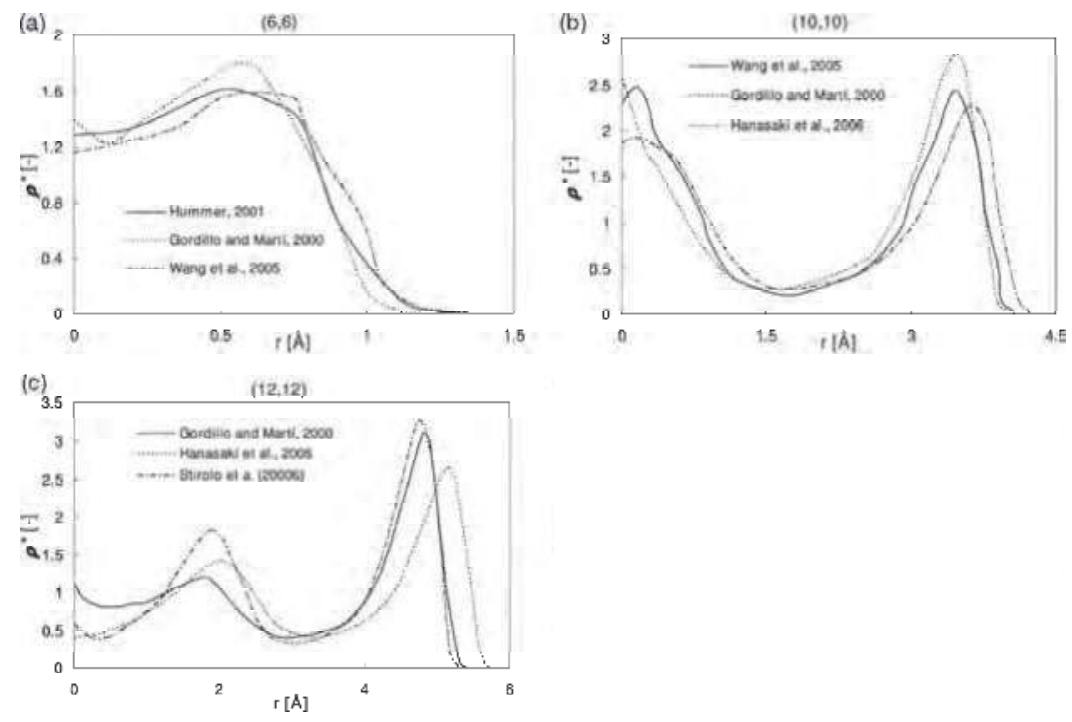

Fig. 2.6. Dimensionless local water density $\left(\rho^{*}\right) \rho /\langle\rho\rangle$, where $\rho$ is the actual and $\langle\rho\rangle$ the total density) inside different nanotubes (Alexiadis and Kassinos 2008): (a) $(6,6),(b)(8,8),(c)$ $(10,10)$. Note that, in $(a, b, c)$ the abscissas represent the distance from the centerline of CNT. The tube is open to a water reservoir as in Hummer et al. (Hummer, Rasaiah et al. 2001) and Hanasaki and Nakatami (Hanasaki and Nakatani 2006), and closed in all the other cases.

\subsection{Water infiltration and fast transport in CNT}

Many researchers have raised a question whether it is possible that the hydrophilic liquids, especially water, can fill in such a narrow hydrophobic channel of CNT and whether there exists a minimum diameter of a CNT to allow spontaneous filling. Aluru and his group (Aluru, Won et al. 2006) carried out MD simulations to point out that the van der Waals interactions between water molecules and carbon atoms are primarily responsible for water filling. Through this process, they could put a limiting size of CNT for water infiltration. For example, water cannot go into a $(5,5)$ SWNT with diameter of $6.9 \AA$ A In addition, Wang et al. (Wang, Zhu et al. 2004) suggested a minimum diameter that allows to form a certain number of water layers $(n)$ is approximately $2\left(\sigma_{C O}+(n-1) \sigma_{O O}\right)$ by employing different sets of 
C-O interaction potentials in their simulation. Washburn (Washburn 1921) provided an explanation of the capillary filling and liquid meniscus dynamics by use of Hagen-Poiseuille equation and Young-Laplace equation. The imbibing length $L$ at time $t$ of a capillary of radius $R$ is given by:

$$
L^{2}=(R \gamma / 2 \mu) t
$$

where $\gamma$ is a liquid/vapor surface tension and $\mu$ is the viscosity of liquid. The Washburn equation (Equation (2.11)) is very useful in describing the marching front of a viscous liquid in capillary tubes as time goes by, while less powerful in explaining the marching motion for non-slip boundary or very short period of time. Indeed, filling narrow CNTs like $(6,6)$ with water molecules is not an ordinary phenomenon because water molecules would have no reason to fill in such a narrow hydrophobic channel of CNT at the first glance. However, both MD simulation and experiments support that water can gain access to narrow nanotubes even though a water molecule may have to lose around two hydrogen bonds, corresponding to ca. $10 \mathrm{kcal} / \mathrm{mol}$. One way of explaining this counterintuitive phenomenon is to compare binding energy distributions of hydrogen bonds among water molecules at bulk and under the CNT nanoconfinement. According to MD simulation (Hummer, Rasaiah et al. 2001), the probability distribution of the hydrogen bonding energy between water molecules becomes narrower under the nanoconfinement than in bulk state as shown in Fig. 2.7.

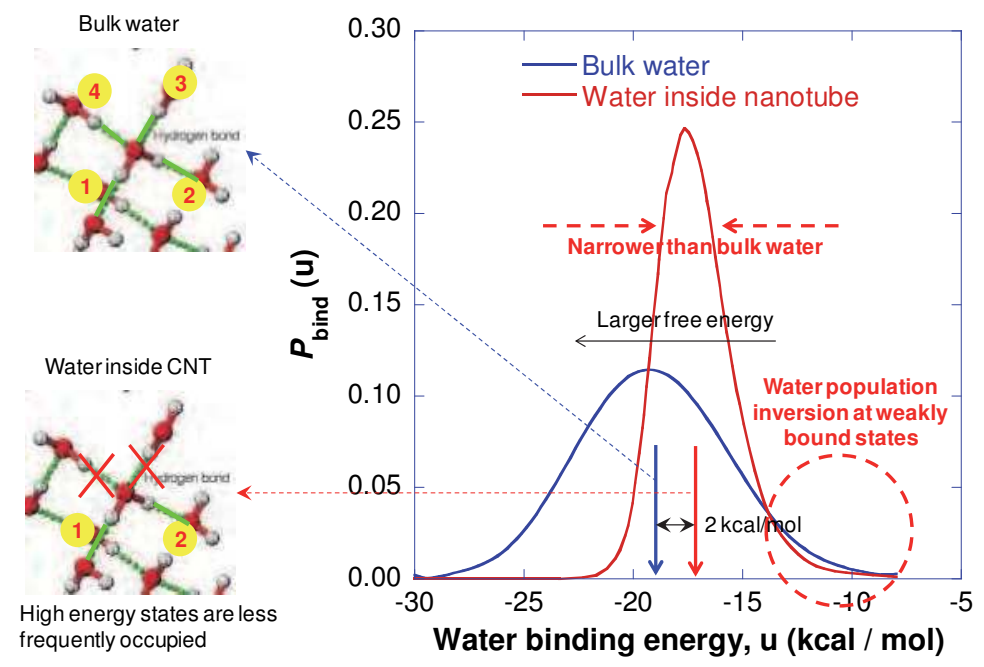

Fig. 2.7. Probability distribution over water binding energy for bulk water (blue) and water inside CNT (red). Two vertical arrows indicate average binding energies. Dotted circle emphasizes the population inversion region, in which weakly bound states are more populated in bulk water. Reconstructed from Hummer et al. (Hummer, Rasaiah et al. 2001).

For water in $\mathrm{CNT}$, this change can shift the mean binding energy value toward a higher one, energetically unfavorable. Even energetic compensation given by van der Waals attraction between water and CNT (ca. $4 \mathrm{kcal} / \mathrm{mol}$ ) is not found sufficient to cover the $10-\mathrm{kcal} / \mathrm{mol}$ 
enthalpic loss. The degeneration in water binding energy distribution, however, can result in a lower population in the high energy region, which can effectively lower down a local excess chemical potential, enthalpo-entropic gain. This finding implies that water molecules can overcome enthalpic penalty and obtain enthalpo-entropic gain to be thermodynamically stable under the CNT nanoconfinement as predicted by Hummer et al. (Hummer, Rasaiah et al. 2001). Subsequently, they pointed out that the water occupancy in CNTs (hydration) is dominated not by how strongly bound the hydrogen bonds are on average but rather by how weakly these bound states are populated. Also, by tuning the water-to-carbon interaction potential, they demonstrated that it is possible to create a transition state in which CNT may be either filled or empty. This intermittent permeation can be described as bi-stability. Zhao et al. (Zhao, Liu et al. 2009) explored a critical pressure barrier associated with infiltration of SWNT employing nonequilibrium molecular dynamics for various temperatures, diameters, and surrounding pressures, assuming that the hydrogen bond is associated with the surface tension of the confined water. Reduced critical pressure barrier makes it easier for water molecules to fill in the tube, which indicates that a small pressure barrier could enlarge the accessible entry area of the nanotube, thereby weakening the hydrogen bond interaction. Their results showed that the magnitude of the critical pressure barrier could become smaller as the temperature increases, the diameter decreases, and loading rate gets higher. Waghe et al. (Waghe, Rasaiah et al. 2002) who carried out the first study on the filling and emptying kinetics of water inside SWNT, calculated the free energy of water molecules transporting inside a $27 \AA$ long $(6,6)$ CNT. They took into account that filling thermodynamics and kinetics depend on sensitivity of the attractive interactions between the nanotube and water molecules which increases with the length of the tubes, and the Gibbs free energy increases until the nanotube is filled completely. After then, additional entry of water molecule makes another hydrogen-bond chain with the initial orientation. If one water molecule would go into CNT, the movement of an additional molecule costs less Gibbs free energy. Finally, this behavior makes water molecules filled completely in CNT and reach the Gibbs free energy minimum. However, filling motion cannot be happened simultaneously from both ends because of the dipolar orientation incompatibility. The water chain can be broken up especially at the end of tube where the direction of the hydrogen bond points outward, which can explain water behaves burst-like motion. The water filling mechanism can be summarized using the Gibbs free energy.

$$
G_{N}=-\ln p(N) / k_{B} T,
$$

where $G_{N}$ is the Gibbs free energy of $N$ water molecules occupying a nanotube. $G_{N}$ is associated with the probability function $p(\mathrm{~N})$ that $\mathrm{N}$ molecules can be found inside $\mathrm{CNT}$.

First, water filling occurs at the end of a nanotube, with hydrogen entering first and dipoles orientation pointing inward CNT

$$
G_{0}=0, G_{1}=\Delta G_{0},
$$

Second, successive entry of water molecules grows the hydrogen-bonded chain with the initial orientation and the chain progresses with all dipoles pointing inward. The Gibbs free energy of introducing one additional molecule to the chain, $\Delta G$, linearly depends on an attraction potential control parameter $(\lambda)$ to be taken account into below

$$
G_{N}=\Delta G_{0}(\lambda)+(N-1) \Delta G(\lambda), \Delta G \propto-\lambda,
$$


Finally, the last water molecule going into CNT may enter from either side of the tube. If $\mathrm{M}$ is defined as the maximum available number of molecules in a single-file chain,

$$
G_{M}=G_{M-1}+\Delta G_{1}(\lambda),
$$

In the meantime, if water indeed enters CNTs spontaneously, what can affect the water transport according to confinements? There still exist different assertions on why carbon nanotubes are fast transporter of water because simulation results are very sensitive to the choice of water models and potential properties and few experimental studies are provided to verify only the fact that the water indeed flows with much larger flow rate than expectations in macro- or micro- scale. However, it is no doubt that the reasons should be classified into water structure that minimizes the energy to move or hydrophobicity that indicates smoothness of the channels. Up to now, it is commonly explained that the fast water transport in CNTs is caused by the hydrophobicity to make a depletion region at the interface occurred. In recent work, Thomas and McGaughey (Thomas and McGaughey 2008) reassessed the fast water transport in CNT by deriving an equation of flow enhancement in CNT from a continuum based, pressure-driven flow model that incorporates the variation of water viscosity and slip length with respect to the CNT diameter.

$$
\begin{gathered}
\varepsilon=\left[1+8 \frac{L_{s}(d)}{d}\right] \frac{\mu_{\text {bulk }}}{\mu(d)}, \\
\mu=\mu_{i} \frac{A_{\text {interface }}}{A_{\text {total cross section }}}+\mu_{\text {bulk }}\left(1-\frac{A_{\text {interface }}}{A_{\text {total cross section }}}\right)[m P a . s], \\
L_{s}=30+\frac{352}{d^{3}}[\mathrm{~nm}],
\end{gathered}
$$

Equation (2.16) shows good agreement with MD simulations, also valid in CNTs with larger diameters than $1.66 \mathrm{~nm}$. The enhancement factor obtained by Holt, Park et al. (Holt 2006) is in proximity to the range this equation can predict. Kalra et al. (Kalra 2003) proposed the single-file water transport in CNTs can be modeled with a one-dimensional random walk theory. From their work, water transport in CNTs should be fast due to nearly frictionless surface and microscopic fluctuations. One-dimensional random walk model is limited to a single-file conduction of water. Joseph and Aluru (Joseph and Aluru 2008) investigated hydrogen bonding configuration of water under different degrees of CNT nanoconfinement via comparison of effects of diverse interatomic potentials by holding nanochannel geometry of 1.6-nm-wide CNT. In this study, they compared four distinct nanoconfinements of water: (1) hydrophobicity and atomic-level smoothness (CNT); (2) hydrophobic and nonatomic-level smoothness (BNNT); (3) hydrophilic and atomic-level smoothness (CNT with interatomic potential of $\mathrm{Si}$ ); (4) hydrophobic and nanoscale roughness (CNT with a several angstrom of step). For the CNT nanoconfinement that is hydrophobic and smooth on the atomic level, water transport showed an enhancement by 3 orders of magnitude compared with Hagen-Poiseuille formalism. The hydrophobic but less smooth nanoconfinement of BNNT also enhanced the flow, but the enhancement was slightly smaller. The hydrophilic and atomic-level smooth nanoconfinement, however, lost a significant amount of flow enhancement. Lastly, the nanoscale roughness provided by connecting two CNTs having 
different diameters negated the flow enhancement effect almost completely. They described that the fast water transport depends primarily on the water configuration caused by the hydrogen bonding nature because the enhancement factor even in a silicon nanotube that has larger friction is still larger than predictions calculated by slip length theory as a number of hydrogen bonds decrease. They also identified a hydrogen-bonding depletion layer near the hydrophobic wall, which is in part responsible for the ultrafast water transport.

Hummer et al. (Hummer, Rasaiah et al. 2001) have presented a single-file water wire that showed the burst-like motion along the nanotube axis because, in their equilibrium MD simulation, rapid conduction of water was driven by thermal fluctuation of water molecules at either open end of CNT. Beckstein and Sansom (Beckstein 2003) have also pointed out that it is not a single-file chain but one-dimensional nanoconfinement that increases the selfdiffusivity of water, thereby supporting bursting motion of water molecules along the tube although water molecules retain the three-dimensional molecular thermal velocity distribution under this confinement. Up to now, there have been several efforts to suggest reasonable equations for comparing and understanding the fast water transport in CNT. Thomas and McGaughey (Thomas and McGaughey 2008) suggested the pressure-driven water transport equation (Equation (2.16)) based on the slip length theory for CNT diameters between 1.7 and $5.0 \mathrm{~nm}$, assuming that the large flow velocity though CNT is mainly caused by the nearly frictionless surface. Their flow enhancement calculation partially overlapped the range of enhancement given by Holt, Park et al. (Holt 2006), and yet did not agree well with Majumder et al. (MajumderMainak, ChopraNitin et al. 2005). This assessment led Thomas and McGaughey to an argument that the flow enhancement measurements should have been smaller, although their model equation has parameters that has not been determined either by experiment or by exact theory, but has rather been determined from their own simulation result. Therefore, the validity of their argument awaits further experimental and theoretical verification. Recently, Falk et al. (Falk, Sedlmeier et al. 2010) reported a strong curvature dependence of the friction of water inside CNT. According to their MD simulation analysis, the friction decreased when water is placed inside narrower CNTs, leading to super lubricity for sub-1-nm CNT and single-file water wiring. When water is placed outside $\mathrm{CNT}$, the friction increased as the curvature gets larger. In a reference case of water on a flat graphene surface, they did not predict any change in friction. The predicted flow enhancement is on the order $10^{3}-10^{4}$ for CNTs with diameters in the range of $1-2 \mathrm{~nm}$ and $10^{1}$ for diameters of the order of $50 \mathrm{~nm}$. Actually, water molecules in CNT is so tightly confined and stratified in the radial direction that they may experience less thermal fluctuation in the radial direction, thereby allowing thermal fluctuation in the axial direction to have a major effect.

Based on the aforementioned results, here we present a summary of the water filling mechanism in Fig. 2.8.

\subsection{Summary}

Up to now, a large number of studies have been carried out on the phenomena and properties of water confined inside CNT. We focused in this chapter on the simulation results of water infiltration of and transport through $\mathrm{CNT}$, and the experimental works for the verification of theories. It is very interesting that water can enter and flow so rapidly in narrow and hydrophobic CNTs. Since 2001, MD simulations have started providing the first answer about these nanoscale phenomena. Researchers pointed out that the water infiltration of narrow CNTs is related to the degeneration of hydrogen bonding energy 
distribution that lowers chemical potential in effect. When water is placed in the CNTs, hydrophobic interaction takes an action to create a depletion region between water and the graphitic wall of CNT, thereby smoothening a potential landscape that water experiences during the transport. Hence, we need to note how the water can enter and fill inside CNTs with thermodynamic equilibrium state. According to several simulation results, one may make a summary of water infiltration, water structure and fast water transport.

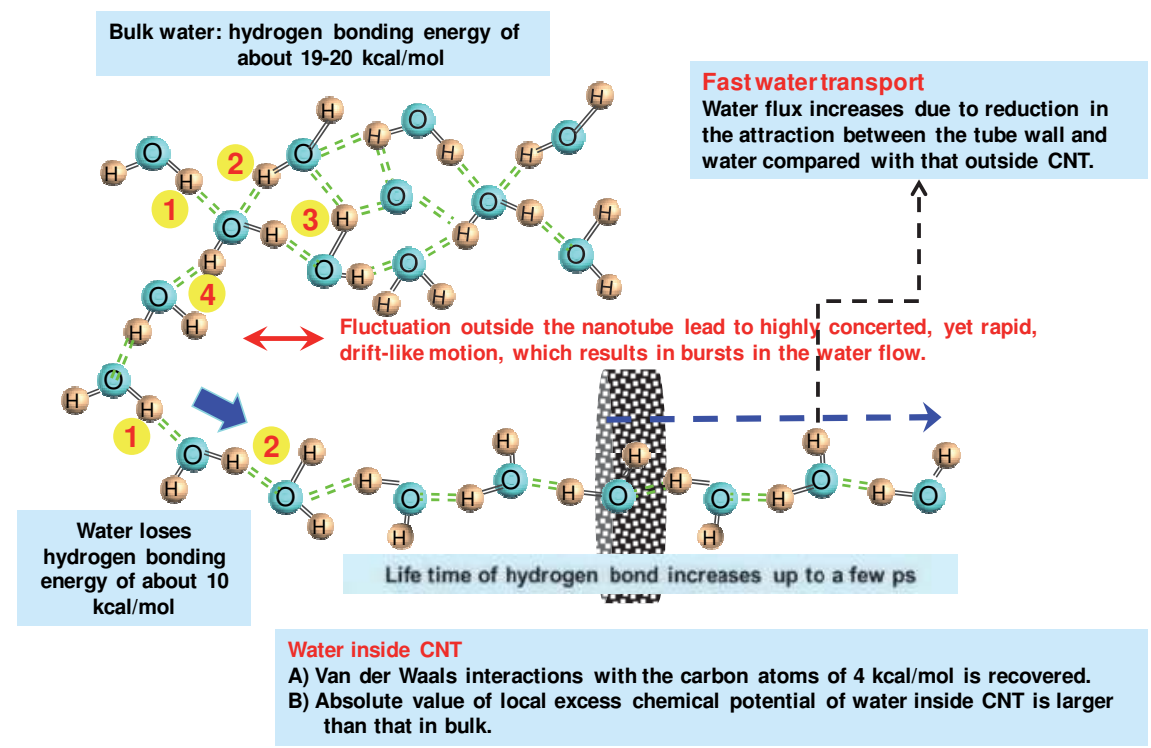

Fig. 2.8. Schematic explaining briefly how water can fill in CNT and water transports fast along CNT.

1. As the CNT diameter increases up to a certain minimum value for hydration, water molecules fill the narrow space rather homogeneously, yet not hydrogen-bonded, as expected for vapor.

2. While the configuration becomes a single-file chain in CNTs with diameter of sub $1 \mathrm{~nm}$, hydrogen bonds form distinct layers as well as a single-file chain in wider carbon nanotubes. In addition, the number of water layers inside CNTs increases from one to two and from two to three sequentially, with the central water column being the preferred position.

3. As the CNT diameter further increases, the maximum radial density shifts slightly from the center toward the wall, and thus the center line is not favored energetically. Moreover, the curvature effect on density weakens so that the radial density distribution seems almost identical to the situation near a planar hydrophobic slab such as grapheme layer, with most of the density near the center line being close to that of bulk water.

4. Once water molecules fill in CNT, the flow velocity profile is far from parabolic derived from the momentum transport mechanism in the continuum based fluid mechanics theory, rather it is more plug-like than parabolic because hydrophobicity weakens the friction and confines its effects to the outermost layer. 
Carbon Nanotube Nanofluidics of water still raises unanswered fundamental questions in comparison with conventional theories even though there are a number of literatures that predict water configuration and flow in CNTs. How can one observe the relationship between the hydrogen bonding (or water configuration) and fluidic properties such as viscosity, density and phase change? Would it be possible to explain the infiltration and transport phenomena in view of an enthalpo-entropic picture?, Can one suggest a new dimensionless number that can well characterize the water transport inside CNT? How can one predict the dependence of CNT hydration on other inherent CNT properties such as nanotube charge and length? Finally, can one make some sorts of validation mapping of simulation results by implementing more experiments in near future before we apply Carbon Nanotube Nanofluidics to any useful engineering technologies?

Taking a look deeper, it would be an interesting and promising work to offer new viewpoints tangential to conventional theory, from which new applications with high efficiency and portability could be developed. Accordingly, if answers about the above questions are found and validated by experiments in the foreseeable future, we may also make one step closer to the description of biological translocation processes such as a biomolecular (DNA, RNA) transport through biological pores that share their dimensions with CNT.

\section{Gas transport}

\subsection{Introduction and overview}

The use of CNT as a membrane pore wall material for gas transport gives an additional and possibly the most significant advantage because the bottom-up process of CNT array synthesis can provide molecularly smooth wall for the gas flows, notwithstanding the fact that the nanochannel size becomes commensurable with the size of the fluid molecules flowing through it. Thus, one may raise a question about the way in which properties of gas inside CNT are affected by diameters, lengths, and tube shapes. Perhaps one may be more concerned about pumping power required for transporting a certain amount of gases (Wang, Gupta et al. 2005). Many fundamental studies in the past several years revealed that the infiltration and emptying mechanisms of water, gases, and ionic liquids possess distinct characteristics from those of bulk phase. Among these discrepancies, gases inside CNT of only a few molecular diameters are well known to diffuse rapidly due to the unique CNT properties such as molecular smoothness and nanoconfinement effect (Skoulidas, Ackerman et al. 2002). Gas molecules can pass nanotube pores by orders of magnitude faster than they pass pores out of other materials of comparable sizes. When gases are confined inside CNT, their properties are quite different from those seen for the bulk fluid. This phenomena is often characterized by the Knudsen number defined as the ratio between the gas mean free path and the characteristic length given by the confinement (Cannon and Hess 2009). Simply speaking, the former length scale represents intermolecular collision-based, momentum and energy exchanges, whereas the latter does momentum and energy exchanges between transporting gas molecules and the substance comprising the confining wall. Indeed, it is very important to understand the diffusive behaviors of gas molecules in CNT because of possible applications such as drug delivery and selective transport (single- or mixed gas separation). Skoulidas et al. (Skoulidas, Ackerman et al. 2002), for the first time, reported that diffusion of light gases through SWNT can excel diffusion through zeolites by orders of magnitude, implying energy efficiency in using $\mathrm{CNT}$ as a gas separation medium. Current 
studies of gas transport inside CNT are focusing on large liquid-solid interfaces for a flow boundary condition analysis (Steitz, Gutberlet et al. 2003) and surface force measurements (Meyer 2005), as evidenced by grazing incidence X-ray scattering by Daillant (Daillant 2009). Nevertheless, both theoretical and experimental studies on the kinetics of gas-liquid-solid interactions with respect to confinement are still missing. One of the primary objectives of this chapter is to explore gas transport under the nanoconfinement. In general, different transport types or flow regimes can be categorized via Knudsen number as shown in Fig. 3.1. In terms of CNT diameter, a Knudsen number larger than 10 from which gas transport in CNT enters a free molecular flow regime can be obtained CNT interior diameters below $6.5 \mathrm{~nm}$. However, one may raise a question whether gas in CNT with diameter of $6.5 \mathrm{~nm}$ has characteristics of transition flow or those of free molecular flow since the classification of flow regimes is not too strict. Therefore, it would be necessary to carry out case studies with respect to CNT diameter.

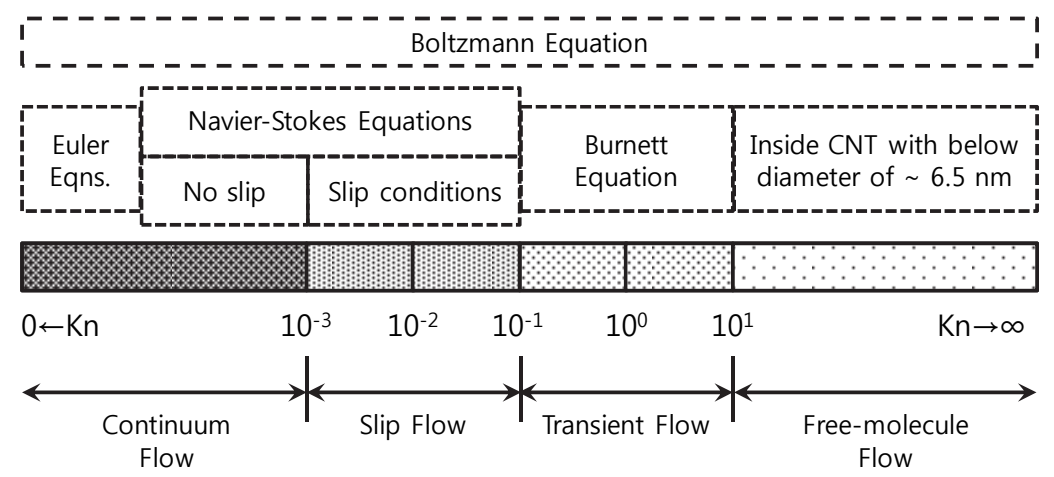

Fig. 3.1. Flow regimes categorized by Knudsen numbers (Roy, Cooper et al. 2005). It should be noted that the classification is not strict and the categorizing Knudsen number between flow regimes may vary, depending on the flow situation.

Meanwhile, Holt, Park et al. (Holt 2006) reported molecular weight selectivity referenced to helium flows, with measuring five hydrocarbon and eight non-hydrocarbon gas flows through the pores of sub-2-nm DWNT, or Knudsen number of ca. 30. They measured gas flow rates of 16 - 120 times larger than a Knudsen diffusion prediction that models free molecular gas transports. Generally, it is collisions between gas molecule and tube wall that may significantly alter the way of describing the micro-nanoscale flows. Two types of collisions are generally classified. One is called a "diffuse collision," or "Knudsen diffusive collision" or "ballistic diffusive collision," wherein a colliding gas molecule thermalizes itself with a wall and randomizes its bouncing velocity whenever it collides with the wall. The source of the velocity randomization may include wall roughness on atomic to mean free path levels and chemical inhomogeneity (Malek and Coppens 2003). The other is a "specular collision," in which a reflecting gas molecule will conserve its incident tangential velocity (momentum) component along a colliding surface. Specular reflection on the wall is sometimes referred to as slippery collision. Generally, rarefied gas collisions may have both diffusive and specular components. One way of incorporating the component of specular collision in transport theories is to introduce as a correction factor a parameter called tangential momentum accommodation coefficient (TMAC or $\sigma_{v}$ ) as a measure of a portion of 
molecules that undergo diffuse reflections. A detailed interpretation of TMAC is well reviewed by Sokhan et al. (Sokhan, Nicholson et al. 2004). Considering the influence of slippery collisions between gas molecules and the pore wall, the Knudsen-Smoluchowski correction model was introduced to experiments for argon and oxygen flows through 10-nm polycarbonate nanopores by Roy et al. (Roy, Cooper et al. 2005). According to the model, the effective transport diffusivity is given by $D=D_{K n}\left(2-\sigma_{v}\right) / \sigma_{v}$, where $D_{K n}$ and $\sigma_{v}$ represent the original Knudsen diffusivity assuming a 100\% diffuse collision and tangential momentum accommodation coefficient, respectively. For $10 \mathrm{~nm}$ polycarbonate membranes, their experiment resulted in TMAC to be 0.28 . In a slip flow or an early transition regime $(\mathrm{Kn} \approx$ 0.4), Arkilic et al. (ARKILIC, BREUER et al. 2001) reported a value of 0.8 for a nitrogen gas on a prime silicon wafer surface. The Knudsen-Smoluchowski diffusion model is a correction model of the Knudsen diffusion model, and in an isothermal free molecular flow regime, the Smoluchowski-corrected flow rate is obtained by multiplying $\left(2-\sigma_{v}\right) / \sigma_{v}$ to the original Knudsen flow formula. More detailed explanation of TMAC will follow in next section. Finally, experimentally measured gas flow through the nanopores can be compared with theoretical predictions useful in the nanometer size regime, e.g. Knudsen diffusion model, the unified flow models, or MD simulations. In this section, we introduce three flow models that describe the behavior of gas species under nanoconfinement, and discuss possibilities and perspectives of the CNT gas transport paths as a passive gas separator.

\subsection{Investigation of transport theories 3.2.1 Consideration of two length scales}

If air molecules distribute themselves evenly in a three-dimensional box, an estimate of their intermolecular spacing can be $n-1 / 3$, where $n$ is a molecular number density at the corresponding thermodynamic conditions. Treating air as an ideal gas, $n=P / k_{\mathrm{B}} T$, where $P$, $T$ and $k_{\mathrm{B}}$ are pressure, temperature and Boltzmann constant, respectively, results in average intermolecular spacing of about $3.4 \mathrm{~nm}$ at room temperature and $1 \mathrm{~atm}$. This spacing is about 10 times larger than molecular sizes of nitrogen and oxygen. In such a dilute medium, most of the momentum and energy transfers occur via intermolecular collisions. In random collisions, the most probable situation is the collision between two molecules possessing distinct thermal velocities. It is not the average gas spacing, but the mean free path that will determine the momentum and energy transfers in gas phase collisions. Estimation of the mean free path at $1 \mathrm{~atm}$ and $300 \mathrm{~K}$ gives a mean free path of $70 \mathrm{~nm}$ for air, which is about 20 times larger than the average molecular spacing and more than 200 times larger than the size of a molecule. If a carbon nanotube of $2 \mathrm{~nm}$ in diameter is placed in the box, air molecules will primarily collide on the external surface. Sometimes a portion of molecules will get into the open ends. Some of the incident molecules will bounce back at the mouth and some will go through and emerge from the other open end. Since the nanotube diameter is much smaller than the air mean free path, molecules inside the nanotube will collide exclusively with the wall, instead of exchanging momentum and energy through intermolecular collisions.

Since collisions induce momentum exchange, the nature of the collisions is important in describing mass transport or flow through a nanoscale channel. Two length scales become important: the mean free path and the channel size. If the mean free path is much smaller than the transverse channel size, momentum transfer will take place most likely through intermolecular collisions and gas kinetic theory may determine the fluid property. This 
type of flow is called macroscale flow. If, on the other hand, the mean free path becomes much larger than the lateral channel size, most of the transport will occur through molecule-to-wall collisions, in which case the dynamics of the transport will be undoubtedly different. Therefore, a comparison between those two length scales enables determination of transport types. Knudsen number $(\mathrm{Kn})$, defined as a ratio of the mean free path to the channel characteristic length is an important parameter considered in this comparison.

\subsubsection{Knudsen diffusion model}

The Knudsen diffusion model is widely used to describe mass transport in the free molecular flow regime. The regime where the diffusion process is dominated by fluidinterface collisions (large Knudsen number) is called the "Knudsen regime" or "rarefied gas state." Knudsen (Steckelmacher 1986) developed a free molecular flow model based on kinetic theory and his experiment of long tubes in vacuum. He proposed a formula which can be used in all flow regimes by combining the Poiseuille flow equation for viscous flow, his model for the free molecular flow and an interpolating function determined by a fit to his experimental data for transition flow, which is called "Knudsen flow model" because the difference between molecular flow and diffusion is not definite at the microscopic level in the free molecular regime. There are two types of diffusion coefficients that are related to mass transfer within the pore: Maxwellian and Knudsen. Knudsen diffusion occurs when the molecules in the fluid collide with the pore walls, while Maxwellian diffusion is also known as bulk diffusion caused by intermolecular collisions. Knudsen diffusion is prominent when the mean free path of the molecules is much larger than the diameter of the pore. The formula in the free molecular gas flow regime is as follows.

$$
J=D_{\mathrm{Kn}} \frac{\Delta P}{R T L}, D_{\mathrm{Kn}}=\frac{d}{3} \sqrt{\frac{8 R T}{\pi M}},
$$

where $J, D_{K n}, P, R, T, L, d$ and $M$ represent a molar flux, Knudsen diffusivity, pressure, universal gas constant, temperature, channel length and diameter, and molecular weight, respectively. The original Knudsen diffusion model, thus Knudsen diffusivity, hypothesizes diffuse reflection as a boundary condition between molecules and channel (pore) wall. The other type is specular collisions, where a reflecting gas molecule will conserve its incident tangential velocity component along a colliding surface, a concept of slippery collision. The rarefied or nanoscale gas collisions may generally have both diffuse and specular components. For instance, The Knudsen number of 0.17 for a nanotube with a diameter of $O(1 \mathrm{~nm})$ means that the mean free path in the nanotube is less than $0.5 \mathrm{~nm}$. Since the mean free path is inversely proportional to the molecular number density or the gas pressure in the nanotube, this small value of the mean free path may mean that the local average number density can be over two orders of magnitude larger than in the STP condition; the mean free path in the STP state gives $\sim 65 \mathrm{~nm}$ for air. In terms of pressure, $\mathrm{Kn}$ of 0.17 for air in sub-2-nm diameter nanotube may be established at about $200 \mathrm{~atm}$ at room temperature. Fig. 3.2 shows the dense molecular packing inside the carbon nanotube Sokhan et al. (Sokhan, Nicholson et al. 2002) have simulated. As stated above, one way of incorporating the component of specular collision in transport theories is to introduce a parameter called tangential momentum accommodation coefficient (TMAC, $\sigma_{v}$ ) as a measure of a portion of molecules that undergo diffuse reflections. 

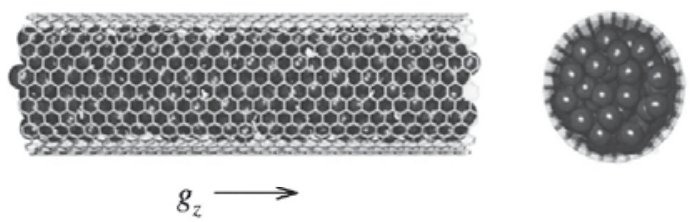

Fig. 3.2. Sketch of the system for MD simulations to investigate gas transport in CNT. An external force is applied along the long axis of CNT. Owing to issues with computational cost, most of today's molecular dynamics simulations of gas transport under CNT nanoconfinement investigate densely packed gas molecules at high pressures (Sokhan, Nicholson et al. 2002).

As the dimensions of the pore diminish, the mean free path $(\lambda)$ becomes larger than the channel dimensions $(d)$ and the transport enters the molecular flow regime. In such situations, where particle-surface collisions dominate over particle-particle collisions, the Knudsen diffusion model is frequently applied. The Knudsen-Smoluchowski diffusion model is a correction model of the Knudsen diffusion model, and in an isothermal free molecular flow regime, the Smoluchowski-corrected flow rate is obtained by multiplying $\left(2-\sigma_{v}\right) / \sigma_{v}$ to the original Knudsen formula. The multiplication factor may contribute to the enhancement factor measurement (Porodnov, Suetin et al. 1974). Maxwell coefficients or TMACs can be estimated from the enhancement factor in by the following relation, where $Q$ and $V_{\mathrm{m}}$ stand for volumetric flow rate and molar volume of a gas species, respectively.

$$
\sigma_{v}=\frac{2}{1+\frac{Q_{\text {experiment }}}{Q_{\mathrm{Kn}}}}=\frac{2}{1+\frac{Q_{\text {experiment }}}{\frac{2}{3} \sqrt{\frac{8 \pi}{M R T}}\left(\frac{d}{2}\right)^{3} V_{m} \frac{\Delta P}{L}}},
$$

The Knudsen diffusion mechanism states that each particle-to-wall collision accompanies complete thermalization in a way that the angle of reflection randomizes fully with respect to the angle of incidence. In that sense, the Knudsen transport mechanism doesn't seem to be somewhat feasible in CNTs as demonstrated by Holt, Park et al. (Holt 2006) because of specular collision that gas molecules are believed to undergo on the molecularly smooth wall of a CNT interior (Maxwell coefficient < 1) (Holt, Noy et al. 2004). The Knudsen diffusion model can still suggest one criterion to compare the flow enhancement of gas molecules through CNT. Roy et al. (Roy, Cooper et al. 2005) reported argon and oxygen flows through a $10 \mathrm{~nm}$ polycarbonate membrane whose rates are an order of magnitude higher than would be predicted by Knudsen diffusion alone. They ascribed the flow enhancement to the rarefied diffusion with slip (KnudsenSmoluchowski diffusion) and obtained the TMAC as small as 0.21 . Through a finite element method modeling with a first-order slip boundary condition, they claimed that the hydrodynamic model may predict Knudsen-like diffusion for Kn up to 10. However, Karniadakis et al. (Karniadakis, Beskok et al. 2005) pointed out that the use of the slip boundary condition to explain the flow enhancement by increasing the total flow rate works well only at the expense of accuracy in the velocity profile. Therefore, the success of the modified hydrodynamic model with appropriate slip boundary condition in high $\mathrm{Kn}$ 
numbers corresponding to transition and free molecular flows needs further investigation with respect to both the total flow rate and the local velocity profile. With a carbon pore membrane with 170 - $235 \mathrm{~nm}$ pores obtained by the chemical vapor deposition of carbon films on the alumina membrane (Miller, Young et al. 2001), Cooper et al. (Cooper, Cruden et al. 2003) saw evidence of modification of the pore surface characteristic from diffuse to diffuse-specular one. They observed gas flow enhancement compared to diffuse Knudsen model and determined TMAC to be 0.52 . Considering the large pore diameter and the fact that the pore became partially oxidized, it is questionable whether the pore may represent fully graphitic carbon nanotube surfaces. Nitrogen flow through fully graphitic MWNTs was first measured by Hinds et al. (Hinds, Chopra et al. 2004). In characterizing their MWNT membranes, they measured nitrogen permeability as $2.6 \mu \mathrm{mol} / \mathrm{m}^{2}-\mathrm{s}-\mathrm{Pa}$, corresponding to $0.59 \mathrm{cc} / \mathrm{cm}^{2}-\mathrm{s}-\mathrm{atm}$. The Knudsen number for the nitrogen flow in their experiment was 9.3, a value that places the gas flow regime on the verge of transition and free molecular flows. They reported that the measured permeability for their membrane was as expected by the Knudsen flow model, implying the TMAC close to 1 for this kind of MWNT. This large value of TMAC of MWNT is somehow unseen in most of the engineered surfaces.

It is the pore number estimation that imparts the single largest uncertainty to the precise determination of TMAC for CNT. As pointed out by Skoulidas et al. (Skoulidas, Sholl et al. 2006), however, the pore density estimation of the MWNT pores shows a certain degree of uncertainty in the later reports: $6 \times 10^{10} \mathrm{~cm}^{-2}$ (Hinds, Chopra et al. 2004), $5 \times 10^{10} \mathrm{~cm}^{-2}$ (Majumder, Chopra et al. 2005), and $1.0-3.4 \times 10^{9} \mathrm{~cm}^{-2}$ as in the supplementary information of (MajumderMainak, ChopraNitin et al. 2005). This order-of-magnitude fluctuation in the number of open pores of MWNT obscures concluding whether carbon nanotubes can enhance gas flows through them or not, which means that the uncertainty on the pore density quantification may also cause a difficulty in comparing the gas flux through a MWNT membrane with the Knudsen model prediction. This uncertainty has its root in part to the nature of "bambooing" or self-blocking of a tube during MWNT synthesis. On the other hand, SWNT and DWNT having smaller inner diameters have not been reported about self-blocking so far.

\subsubsection{Unified flow model}

The Knudsen diffusivities are always lower than experimental results because it is assumed that every collision leads to diffusion itself, therefore it only holds when the TMAC becomes closer to untity. While, the unified gas flow model may successfully predict the flow regime with high Knudsen numbers in association with nanoscale conduits. The unified gas flow model, considering wall slip and rarefaction effects, can predict higher volumetric flow rates than the free molecular flow theory of Knudsen diffusion. Beskok et al. (Beskok and Karniadakis 1999) proposed a theoretical model called the unified gas flow model that is useful in both the transitional and free molecular regimes. The model assumes that the velocity profile is a shifted parabola towards the main flow direction. For rarefied flow, they compared their model with a solution obtained from the linearized Boltzmann equations (Loyalka and Hamoodi 1990) in order to verify agreement with the linear Boltzmann solution in the Knudsen number range between 0.1 and 10 and to determine the general slip coefficient of their model, $b$, to be -1 . In the unified gas flow model, the flow velocity profile is given as follows. 


$$
U^{*}(r, \mathrm{Kn})=\frac{U(z, r)}{\bar{U}(z)}=\frac{-\left(\frac{r}{d / 2}\right)^{2}+2 \frac{\mathrm{Kn}}{1+\mathrm{Kn}}+1}{\frac{1}{2}+2 \frac{\mathrm{Kn}}{1+\mathrm{Kn}}},
$$

where $r$ and $z$ are the radial and axial coordinates, respectively. In the limit of macroscale continuum flow $(\mathrm{Kn} \rightarrow 0)$, the model converges to a Poiseuille flow profile, or $U^{*}=2[1-(r /(d / 2))]^{2}$. The shape of the profile is a parabola shifted in the axial direction by $2 \mathrm{Kn} /(1+\mathrm{Kn})$, with a shrinkage of the maximum velocity in order to meet the mass continuity requirement. In Equation (3.3), $\mathrm{Kn}$ is a local Knudsen number. Meanwhile, the volumetric flow in a pipe can also be obtained by integrating the velocity equation derived from Navier-Stokes equation with Maxwell's slip boundary condition over the tube cross section.

$$
Q=-\frac{\pi(d / 2)^{4}}{8 \mu_{o}} \frac{d P}{d x}(1+\alpha \mathrm{Kn})\left[1+\frac{4 \mathrm{Kn}}{1+\mathrm{Kn}}\right],
$$

In Equation (3.4), $\mu_{o}$ is a bulk dynamic viscosity and $\alpha$ is the only model parameter accounting for the rarefaction or confinement effect.

$$
\alpha=\alpha_{o} \frac{2}{\pi} \tan ^{-1}\left(\alpha_{1} \mathrm{Kn}^{\beta}\right), \alpha_{o}=\left.\frac{64}{3 \pi\left(1-\frac{b}{4}\right)}\right|_{b=-1}=1.358,
$$

where $\alpha_{1}$ and $\beta$ are 4.0 and 0.4, respectively. In Equations (3.4) and (3.5), Kn is a global Knudsen number and usually determined at the average pressure between upstream and downstream. The rarefaction coefficient $(1+a \mathrm{Kn})$ in Equation (3.4) accounts for a hybrid length scale combining the tube diameter and the mean free path, giving variation to the dynamic viscosity. The concept of the variable diffusion coefficient model (Beskok, Karniadakis et al. 1996) is shown below.

$$
\mu \approx \rho \bar{v}_{t h}\left[\frac{1}{\frac{1}{d}+\frac{1}{\lambda}}\right]=\rho \bar{v}_{t h} \lambda\left[\frac{1}{1+\mathrm{Kn}}\right],
$$

The length scale used in determining the dynamic viscosity in kinetic theory is the mean free path. As Kn increases and the flow enters the transition regime, both the channel dimension and the mean free path become comparable. According to Equation (3.6), the fluid viscosity is determined by the combination of these two lengths. In the molecular viewpoint, as $\mathrm{Kn}$ increases the entity a gas molecule collides with will change from other molecules to either the wall surface or other molecules. Thus, the modified viscosity, called the generalized diffusion coefficient, is expressed as follows.

$$
\mu(\mathrm{Kn})=\mu_{o}\left[\frac{1}{1+\mathrm{Kn}}\right]
$$


Equations (3.6) and (3.7) were proposed by intuition (Beskok, Karniadakis et al. 1996; Beskok and Karniadakis 1999). Further study revealed that the rarefaction coefficient further generalizes the variable diffusion coefficient by dividing the bulk dynamic viscosity by $(1+a \mathrm{Kn})$ instead of $(1+\mathrm{Kn})$. Park (Park 2007) reported that the air flow rates through all three DWNT membranes are higher than the unified model prediction by an order of magnitude, while the flow rates through MWNT and PC membranes are within the order of magnitude of the unified flow model prediction. However, they also had a question whether the unified gas flow model applies in the MWNT case or not due to the uncertainty in the open pore density of MWNT membranes, although relatively higher gas flux than predictions was measured.

\subsubsection{Molecular dynamics simulations}

Molecular dynamics (MD) simulations have also found that the gas molecular flux may be more than three orders of magnitude faster in carbon nanotubes than in zeolites. According to their MD simulations, transport diffusivities of light gases such as hydrogen and methane are much higher through SWNTs than in zeolites. The diffusivity reached almost the same level as that of the bulk diffusivity of a gas $\left(O\left(10^{-1} \mathrm{~cm}^{2} / \mathrm{s}\right)\right)$ although the transport diffusivity of gases through zeolites are on the order of $10^{-9}-10^{-10} \mathrm{~cm}^{2} / \mathrm{s}$. Depending on the nanotube diameter, the diffusivity was as large as $10 \mathrm{~cm}^{2} / \mathrm{s}$ for hydrogen. Skoulidas et al. asserted that the large transport diffusivities is caused by intrinsic smoothness of the rolled graphene wall, instead of the details of the molecule-nanotube interaction potentials, thereby the chirality of nanotube doesn't influence on the diffusivity (Skoulidas, Ackerman et al. 2002). In the later analysis (Skoulidas, Sholl et al. 2006), they presented a detailed picture on the behavior of gas molecules inside a SWNT by displaying a density profile and molecular trajectories as shown in Fig. 3.3. At atmospheric pressure, most gas molecules adsorb on the tube wall and make a tubular structure, or a gas nanotube. Unlike water molecules, the gases do not have strong hydrogen bonds among one another, and thus the gas nanotube does not move collectively. Perhaps the right picture is that the gas molecules diffuse downstream, or in the direction toward a lower potential, following the surface of this gas nanotube. The pressure-dependent adsorption gradient, determined by the gas adsorption isotherm assuming local equilibrium, will drive surface diffusion. At the same time, as shown in Fig. 3.3(b), there exist collisions between the wall and traveling gas molecules in the span-wise direction, in which wall smoothness at a molecular level may augment the contribution of specular reflections to the gas transport. The combination of surface diffusion and Knudsen-Smoluchowski diffusion denoted as a configurational diffusion by Skoulidas et al. (Skoulidas, Ackerman et al. 2002) could describe the molecular level gas transport at pressures of the order of 1 bar. Therefore, two different length scales may be considered, one is the surface interaction length scale and the other the tube diameter. The former will describe the trapping of a gas molecule in a gas nanotube near the inner surface of CNT, thereby making this length scale closer to the van der Waals potential well width which is usually between 0.3 and $0.4 \mathrm{~nm}$ for gas species and carbon atoms. The latter will explain the motion of a free molecule traveling across the tube diameter and will be related to the Knudsen-Smoluchowski diffusion. As the pressure increases and more gas molecules can enter the CNT, another gas nanotube could form on top of the first layer. It is the long range van der Waals attraction potential that could enable the formation of these gas nanotubes. The more gas nanotubes form, the more they shield the attraction from the wall. At much higher pressure, gas molecules in the middle of CNT would rather flow with a 
form of chunk than with additional layers as shown in Fig. 3.3(A). According to Skoulidas et al. (Skoulidas, Ackerman et al. 2002; Skoulidas, Sholl et al. 2006), the transport diffusivities are nearly inversely proportional to the CNT diameters. As the radius of a CNT decreases, the gas nanotubes will dominate the configuration of the gas molecules. The layering effect of gas nanotubes seems to play a significant role in gas transport in small-diameter CNTs. They also pointed out that any source on the surface: i.e. a defect that breaks the molecular smoothness of the molecule-CNT potential energy surface may have an adverse influence on the otherwise large transport diffusivity. Sokhan et al. (Sokhan, Nicholson et al. 2001; Sokhan, Nicholson et al. 2002; Sokhan, Nicholson et al. 2004), through a series of MD simulations, have agreed to Skoulidas et al. (Skoulidas, Sholl et al. 2006). They simulated both an atomic network of carbons and a smooth imaginary surface with a given slip boundary condition. Through the simulations, they determined Maxwell's coefficient or tangential momentum accommodation coefficient (TMAC) of the order of 10-3 (Sokhan, Nicholson et al. 2004). This TMAC value means that statistically only $0.1 \%$ of gas molecules in the nanotube are thermalized by the wall and randomize their reflection velocities. In other words, the wall may thermalize a colliding gas molecule with $0.1 \%$ of probability, on average. Based on the high loading and low TMAC values, one can imagine a collective motion of the chunk of molecules, similar to the motion of a liquid plug on a slippery wall. Since a large number of gas molecules were loaded in their simulation domain, their nanotubes were densely packed as seen in Fig. 3.2. The reported Knudsen number is, therefore, less than 0.17 , which places the gas flow in the slip flow regime. They mentioned that, when the fluid density decreases, the fluid-solid collisions begin to dominate molecular collisions.

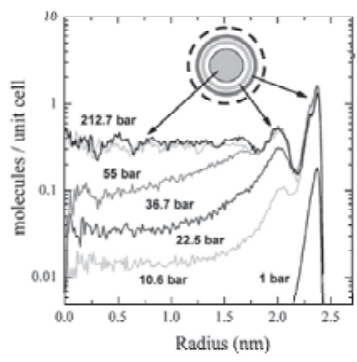

(a)

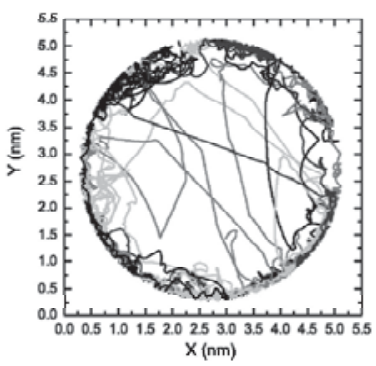

(b)

Fig. 3.3. (a) Density profiles for $\mathrm{CO}_{2}$ adsorbed in a $(40,40) \mathrm{CNT}$ as a function of the bulk gas pressure. (b) Trajectories of $\mathrm{CO}_{2}$ in a $(40,40) \mathrm{CNT}$ at $298 \mathrm{~K}$ and 1 bar (Skoulidas, Sholl et al. 2006).

\subsection{Flow enhancement of single component}

Similar to water transport inside CNT, the gas is known to flow faster than the prediction of the Knudsen diffusion flow through CNT, which can make CNT membranes a potentially high flux and high selectivity medium. Skoulidas et al. (Skoulidas, Ackerman et al. 2002) showed that the potential energy barrier for axial translation of $\mathrm{CH}_{4}$ inside a $(10,10)$ SWNT $\left(0.02 k_{\mathrm{B}} T_{\text {room }}\right)$ is much less than that in zeolite ZSM-12 $\left(1.7 k_{\mathrm{B}} T_{\text {room }}\right)$. The difference in energy barrier can lead to provision of a much smoother energy landscape to the transporting gas molecules under the CNT nanoconfinement. Additionally, many results from MD 
simulations claim that the smoothness of the graphitic wall and relatively weak interaction between gas molecules and inner surface can allow for the fast gas transport. The most common diffusion mechanisms known as activation diffusion, surface diffusion, and Knudsen diffusion, usually dominate in small pores $(d<3 \AA)$, medium pores $(3 \AA<d<10$ $\AA$ ) and large pores $(10 \AA<d<500 \AA$ ) for light gases, respectively (Washburn 1921; Sokhan, Nicholson et al. 2004).

Sholl and Johnson (Sholl and Johnson 2006) reported three orders of magnitude higher gas flux per a pore in CNTs than in zeolites with equivalent pore sizes. In this study, especially, light gases such as hydrogen and methane have almost same diffusivities as that of bulk state. They also showed a motion of gas molecules over the cross section of a SWNT by use of a molecular density and trajectories. According to their result, gas transport through CNTs takes place through predominantly specular collisions between molecule and tube wall, and therefore most of gas molecules flow fast along the CNT. Also, they suggested another transport mechanism that the gas molecules move through CNT with the surface diffusion since some molecules can be adsorbed on the tube walls. Other transport mechanisms were also proposed that emphasize transfer limitations at the tube entrance by Verweij et al. (Verweij, Schillo et al. 2007). They found the efficient transport in the tubes makes the kinetics of surface diffusion becomes more important. Miranda et al. (Miranda, Short et al. 2009) argued that conductance of gas molecules can counter-intuitively decrease as the inlet pressure increases due to the flow impedance given by the tube wall, and asserted that the decreasing sensitivity becomes smaller by showing the experimental results using a direct coupled CNT membrane to a mass spectroscopy. In a CNT membrane, conductance of all the gases used for experiments decreased with increasing total pressure as shown in Fig. 3.4. In other words, the gas flow resistance through CNT grows generally with increasing pressure but at different degrees among gases in terms of the pressure sensitivity of flow resistance. For several gas species, the slope of an increase in the gas flow resistance with respect to pressure, called pressure sensitivity, lies in a descending order: $\mathrm{N}_{2}$ $>\mathrm{CH}_{4}>\mathrm{O}_{2}>\mathrm{Ar}>\mathrm{CO}_{2}$. According to Miranda et al.'s explanation, flow conductance of a CNT membrane decreases with increasing pressure because of interfacial resistance as well as molecular weight. This result was supported and explained in detail by Cannon and Hess (Cannon and Hess 2009). Through a non-equilibrium MD simulation, they reported that the gas flux can be highly influenced by the potential depth at the center of the nanotube as well as by the pore size, and therefore a structure without any defects attributes to higher flow rates. Moreover, the highest gas flux was obtained in the largest nanotubes above a certain back pressure, since the average kinetic energy of a gas molecule exceeds threshold energy for overcoming the interaction between molecules and tube wall. According to their finding, a higher back-pressure can cause a lower flux, and carbon interaction strength can play an important role in separation of gas molecules.

Holt, Park et al. (Holt 2006) have measured the bulk flow rate of air through a DWNT membrane under a pressure gradient of one bar. With observing and estimating the number of pore of the tube by using TEM, they compared the experimental results with other simulations. They concluded that the measured gas flow rates are about one hundred times larger than predictions of the Knudsen diffusion model (see Fig. 3.5(a)). Although their experiment could not reveal the definite reason of the ultrafast flow, the agreement between MD simulations supports that collisions between gas molecules and tube wall shift from diffusive to specular collision under the sub-2-nm CNT nanoconfinement (Skoulidas, 
Ackerman et al. 2002). They also found out that non hydrocarbon gas species follow Knudsen scaling in which enhanced permeabilities of different gas species scale one another to the inverse square root of molecular weight. In addition, they demonstrated one possible route to hydrocarbon gas separation by use of hydrocarbon's deviation from the Knudsen scaling in sub-2-nm DWNT membranes, perhaps caused by additional surface adsorption and diffusion along the surface as shown in Fig. 3.5(b). Mi et al. (Mi, Lin et al. 2007) also fabricated vertically aligned MWNT membranes using CNTs on the $\mathrm{R}_{-} \mathrm{Al}_{2} \mathrm{O}_{3}$ disk. Although this membrane shows a lower value of areal pore density as compared to SWNT or DWNT membranes, their gas permeability is also inversely proportional to the squared root of the molecular weight. Diffusivities of gases in their study are about four times larger than the Knudsen model prediction. Fedorov and Sadreev (Fedorov and Sadreev 2009) investigated the influence of tube diameter and temperature on the self-diffusion of the hydrogen thermo-activated inside three kinds of narrow nanotubes using DFT calculation. They presented that the potential profile is different between $(3,3)$ and $(6,0)$ nanotubes although the diameters are comparable to each other, indicating that hydrogen transport can vary with respect to a CNT chirality because CNTs with different chiralities could induce distinct phonon interaction, or thermal fluctuation, on the tube walls. In addition, the hydrogen density inside CNT was observed to shift in the direction of descending temperature as shown in Fig. 3.6. Accordingly, the authors proposed that it is possible to realize the molecular pump driven by a thermal gradient.

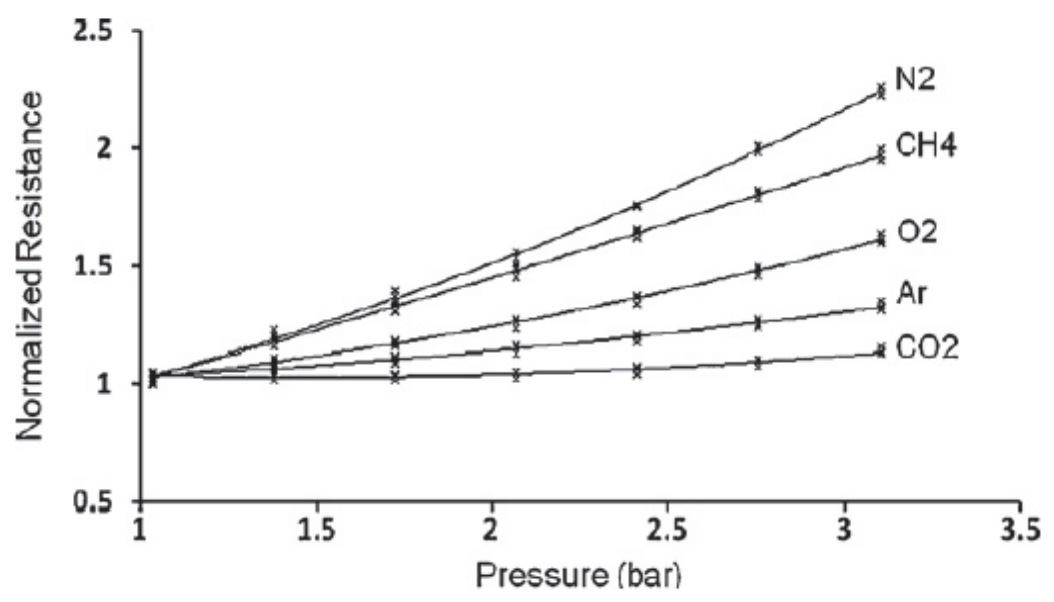

Fig. 3.4. Normalized transport resistance of each gas component in CNT with increasing pressure (Miranda, Short et al. 2009). 


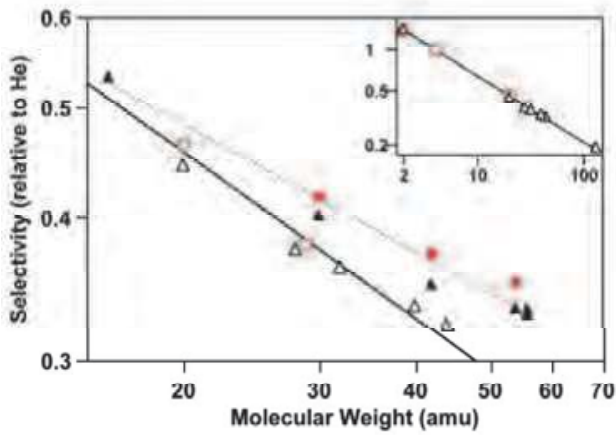

(a)

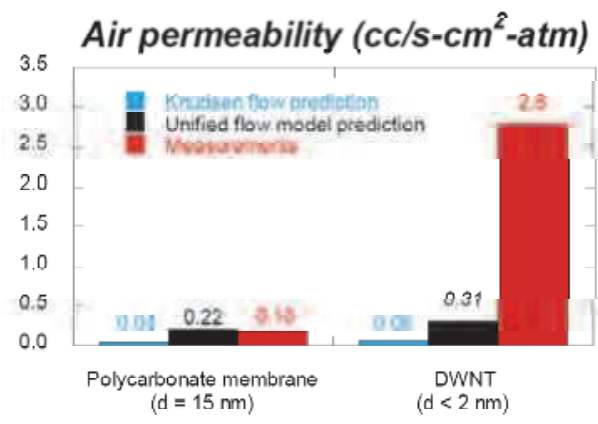

(b)

Fig. 3.5. (a) Gas selectivity (defined as permeability relative to that of He) for sub-2-nm DWNT (triangle) and MWNT (circules) membranes. (b) Comparison of air permeabilities between DWNT and polycarbonate membranes. The average DWNT-membrane permeability is compared with theoretical predictions by Knudsen diffusion model and the unified flow model. DWNT-membrane shows an order of magnitude higher permeability compared to the model predictions, while polycarbonate membrane lies at the same level as them (Holt 2006).

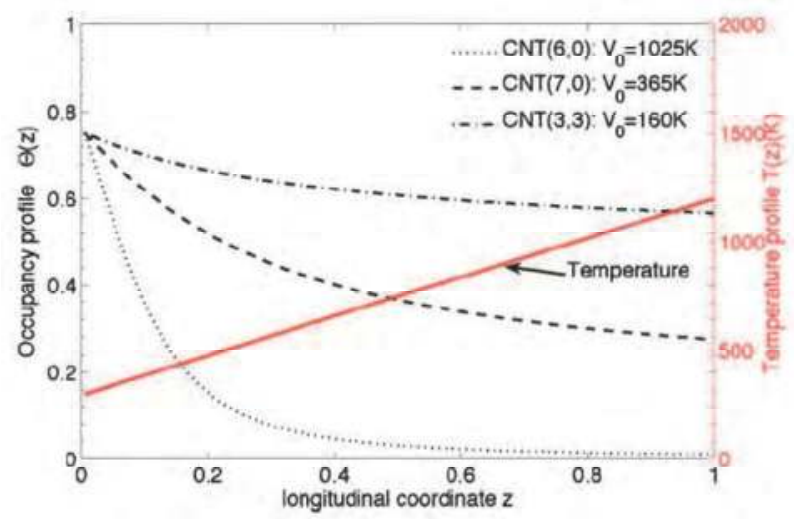

Fig. 3.6. Equilibrium distribution of $\mathrm{H}_{2}$ molecule occupancies $\Theta(\mathrm{z})$ along SWNT and temperature profile T(z) (Fedorov and Sadreev 2009).

Another interesting effect on the gas diffusion is the tube flexibility. Chen et al. (Wu, Sou et al. 2006) observed a distinct influence of tube flexibility on the transport diffusion of single gases and claimed that the influence of tube flexibility on diffusion in the wall-fluid collision dominated the Knudsen regime. The following work done by Jakobtorweihen et al. (Jakobtorweihen, Lowe et al. 2007) showed that CNT flexibility can be very important for the diffusion of rarefied gases. They concluded that the influence of tube flexibility on transport diffusion is stronger for the gas molecule with longer chains. The influence of tube flexibility on the diffusivity of chain-molecules such as methane, ethane, propane, butane, pentane and hexane increases as the chain length of a molecule gets longer, since the longer 
chain molecules have a higher probability to collide with the wall whereas the shorter chain molecules hardly collide. For example, CNT flexibility has a limited influence on the rotational motion for butane whereas the hexane rotational motion is merely influenced by it. Additionally, it has been suggested early to tailor the pore size of membrane by tuning the transport properties (Walther, Jaffe et al. 2001). Thorntona et al. (Thornton, Hilder et al. 2009) suggested a way to distinguish the gas diffusion regime occurring in CNT for different diameters, shapes, and compositions by using a mathematical modeling employing the Lennard-Jones interactions between the gas molecule and the pore wall, which is well matched with the transport of light gases such as $\mathrm{He}, \mathrm{H}_{2}, \mathrm{CO}_{2}, \mathrm{O}_{2}, \mathrm{~N}_{2}$ and $\mathrm{CH}_{4}$. They also showed a minimum diameter for barrier-free transport, a minimum diameter for Knudsen diffusion, and an intermediate region in which an accelerated entering velocity is influenced by the attractive van der Waals forces, in order to provide a theoretical determination of membrane pore sizes, new guidelines to design a CNT membrane for desired separation.

\subsection{Transport in separation applications}

The last decade has seen many efforts to mitigate carbon dioxide emission and reduce the aftermath of global warming by applying lots of chemical reactions to an exhaust gas. Nowadays, the major production cycles consume as much as $40-50 \%$ of the total energy use only for separations, often carried out by inefficient thermally driven separation processes, therefore it became more important to find a new technology that can increase the efficiency and reduce the operating expenditure. Recently, separation of a single species or mixture gases such as oxygen or nitrogen from air using unique characteristics of CNT has attracted much attention. Molecular dynamics (MD) simulations are investigating the idea of gas transport inside SWNT. These simulations reported that the gas transport inside CNT is orders of magnitude faster than inside any other materials with the same pore dimension because the CNT walls have much smoother surface. Indeed, Holt, Park et al. (Holt 2006) who have fabricated the first membranes from aligned SWNT and DWNT showed that CNT membranes do not only possess ultrahigh fluxes but a certain level of single-component selectivity as well. It is noteworthy that the hydrocarbon selectivity among the single components would be more pronounced for practical gas separation problems where mixtures are involved. Since this pioneering work, a number of simulations have noted that CNT may have such amazing characteristics when the CNT membranes are used for gas separations. For example, SWNTs having diameters of $4-12 \AA$ as membrane pores can promise not only fast transport rates but also size-selectivity for a gas mixture $(\mathrm{Wu}$, Sou et al. 2006). Jakobtorweihen et al.'s study on the relationship between SWNT flexibility and nalkane diffusion (Jakobtorweihen, Lowe et al. 2007) has found out that Knudsen diffusivities can predict slower transport for molecules with chain (n-alkanes) or mixtures, since Knudsen diffusion assumes all collisions are diffuse.

Kim and colleagues produced mixed matrix SWNT membranes based on polysulfone (Kim, Jinschek et al. 2007). The authors investigated transport of $\mathrm{CO}_{2} / \mathrm{CH}_{4}$ mixtures experimentally through CNT membranes, and reported that mixed diffusion seems to indicate the presence of transport resistances for molecules entering and leaving the nanotube ends. Following a study on the separation of a gas mixture through CNT carried out by Chen and Sholl (Chen and Sholl 2006), they showed that the selectivity through the SWNT membranes for $\mathrm{CH}_{4} / \mathrm{H}_{2}$ mixture gas is dominated by the preferential adsorption of $\mathrm{CH}_{4}$ relative to $\mathrm{H}_{2}$ in the nanotubes. This preferential adsorption can induce partial filtration of $\mathrm{CH}_{4}$ from the gas mixture to 
present (adsorption) $\mathrm{CH}_{4}$ selectivity ranging from 10 to 35 , depending on the gas pressure. The surface diffusion resistance of molecules moving along the pores increases as the temperature becomes lower or the adsorption strength of the gas molecules inside CNT increases. $\mathrm{CH}_{4}$ in SWNT could also diffuse faster than other light gases such as $\mathrm{Ar}, \mathrm{Ne}, \mathrm{N}_{2}$, or $\mathrm{CO}_{2}$, and the transport mechanism depends weakly on CNT diameter (Wu, Sou et al. 2006), later supported by the work of Newsome and Sholl (Newsome and Sholl 2006). Calculation of surface diffusion resistances for $\mathrm{CH}_{4}$ in a $(20,0)$ SWNT at room temperature revealed that surface diffusion resistances accounting for pore entrance and exit of molecules would be larger for strongly adsorbing species such as n-alkanes than for $\mathrm{CH}_{4}$. This result presents one possibility of separation between $\mathrm{CH}_{4}$ and n-alkanes because stronger adsorption of n-alkanes would expectedly undergo more difficulties in permeating a CNT membrane via the surface diffusion mechanism. Importantly, it is pointed out that surface resistances could be negligible for long CNTs as 5-10 mm but can become significant for sub-micrometer long CNTs.

Separation of a nitrogen-oxygen gas mixture by SWNT was also examined by Arora and Sandler (Arora and Sandler 2006) for various nanotube diameters, temperatures, and pressures, after Wang et al. (Wang, Kim et al. 2005) reported that the nitrogen adsorption energy can increase as the nanotube diameter narrows toward a molecular length scale. They found out that the selectivity of a nitrogen-oxygen gas mixture depends strongly on the nanotube diameters, temperature, and molecular structure. They also reported that the large gas permeability in SWNT has not been merely observed for pure components such as individual nitrogen and oxygen but also for the gas mixture, adding that SWNT has a benign kinetic selectivity for separating of air and the kinks of SWNT could be used for a molecular sieve (Wei, Vajtai et al. 2002). Recently, Kowalczyk et al. (Kowalczyk, Brualla et al. 2007) reported a possibility of storing and separating hydrogen/methane mixtures at room temperatures for SWNT with pore diameters ranging between 0.6 and $2.4 \mathrm{~nm}$. Neither of hydrogen nor methane could enter CNT as narrow as $0.6 \mathrm{~nm}$. When the diameter grows to $0.8 \mathrm{~nm}$, methane could adsorb on the CNT interior. Hydrogen showed selective adsorption and storage capability by forming a bulk gas in a 0.7-nm-wide CNT. Although all of these predictions could not be verified experimentally in the extreme conditions of the simulations owing to the difficulty in fabrication, they provide an understanding of the mechanism responsible for the separation of homogenous or mixture gases by CNT pores, rendering the CNT membrane an option for a molecular sieve with improved selectivity and permeability. However, far more experiments should be carried out to gain better insights about static and dynamic mechanisms of gas separation that keeps fast transport and high selectivity. Qiao et al. (Qiao, Cao et al. 2007) investigated the role of dissolved gases by simulating CNTs of diameters between $1.4 \mathrm{~nm}$ and $5.4 \mathrm{~nm}$. As shown in Fig. 3.7, $\mathrm{CO}_{2}$ gas molecules form a cluster trapped in between water layers of a narrow CNT, while they stay dissolved in water in a wide CNT. They also observed that a single gas molecule can help infiltration and emptying of liquid only in the narrow CNT under pulsating pressures of 200-1000 atm. From this result, it is inferable that gas molecules inside narrow, hydrated CNT could be a driving force for wetting or dewetting of the CNT. Accordingly, further efforts to obtain the high flux and selectivity of CNT membranes by combinational use of gas and liquid can serve as one of the interesting options for gas separation. Finally, breakthroughs in gas separation technology would be made in an accelerating fashion by a hybrid merge of CNT membrane technology enabled by the Carbon Nanotube Nanofluidics' preliminary achievements to industrial techniques already used and under development. 


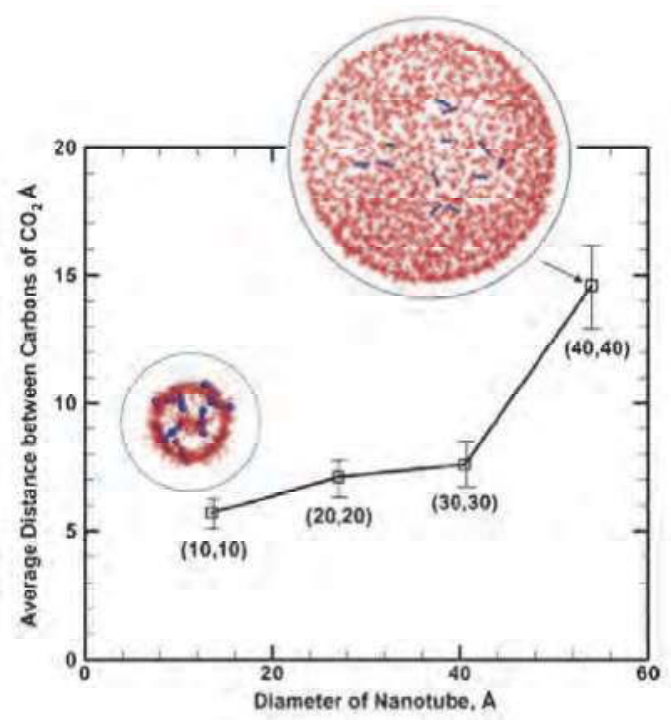

Fig. 3.7. Average distance between carbon atoms of $\mathrm{CO}_{2}$ molecules within hydrated CNTs of different diameters (Qiao, Cao et al. 2007).

\subsection{Summary}

Gas transport in CNT can undergo specular collisions with the interior CNT wall to maintain their forward momentum in a different way from Knudsen diffusion. This mechanism is particularly useful for separation of single- or mixture gas molecules. Recent years after Holt, Park et al. (Holt 2006) have seen lots of experimental studies carried out to investigate somewhat counter-intuitive phenomena about gas transport behavior inside CNT and to validate predictions of MD simulations.

Although the complete fundamental mechanisms of gas transport for various operating conditions are not unveiled until now, it is of no doubt that, in the foreseeable future, a rational design and an improved fabrication scheme of CNT membranes will emerge and lead to the development of a technology of gas separation and purification that focuses on reduction of energy and capital consumptions. We believe it will be possible because of the unique properties of large permeability and of the possibility of high selectivity that CNT membrane can pose. We also stress that the manufacturing efforts of developing large-scale CNT membranes should continue to be exerted, in parallel with the investigation of the fundamental science, in order for the CNT membrane technology to gain economic viability and market acceptance.

\section{Ionic solution in CNT}

\subsection{Introduction and overview}

Aqueous solutions of ions partake in many biological processes. Depending on applications, ion-water systems could be assigned three different categories; ion transport system (when ions in CNT are desirable), ion selectivity system (when ions are selectively desirable in $\mathrm{CNT}$ ) and ion exclusion system (when ions are undesirable in CNT). One of the most 
important cases of ion exclusion is water purification for human consumption and for other purposes including medical, chemical, and industrial uses. Most of the modern interests in desalination/demineralization are centered on development of cost-effective ways of providing potable water in which most of the dissolved salts are ionic in nature. Although membrane techniques such as reverse osmosis (RO) (Suk, Raghunathan et al. 2008), electrodialysis (ED) and membrane distillation (MD) for desalination are widely employed all over the world, the development of more efficient membrane materials that can separate salt from water draws critical attention as it has potential of widening fresh water availability by making desalination cheaper.

The fast water transport in CNT discussed in the previous section and pore sizes commensurable with the ions' own dimensions are rendering use of CNTs a very promising option for the removal of undesirable chemicals, materials, and biological contaminants from contaminated water sources. Using molecular dynamics simulations, Corry et al. (Corry 2007) reported that CNT membranes with sub-nanometer-wide pores can provide significantly higher performance when used in a reverse osmosis-type desalination. According to the simulation, CNTs of $(5,5)$ and $(6,6)$ chiralities could reject $100 \%$ of salt, a 680-to-1100-fold improvement than commercial membranes such as FILMTEC SW30HR-380 used for reverse osmosis. This expected achievement was attributed to the energy barriers at the entrance and exit of such narrow tubes that can control the permeation of ions into CNT pores. The detailed mechanism is related to the energy cost required for removal of water hydration shell surrounding ion. For water entry to the same CNT pore, in turn, it is stability of the remaining hydrogen bonds of water molecules inside CNT that minimizes the energy cost spent for the entry.

Likewise, ion channels can regulate many functions in human body such as transfer of electrical impulses to the nerves, hormones secretion, muscle system control and many others. Diffusion of ions through the bio channels is supposed to be a simple physical process. Characteristics of ionic transport in CNT could be used as simplified theoretical and experimental models for the transmembrane protein studies for inorganic ion flux across cell membranes in a way of improving our understanding of how ions get into and out of cells. This understanding would lead us to bio medical solutions of many human deceases caused primarily by ion channel dysfunction. Selectivity hypothesis of $\mathrm{K}^{+}$from $\mathrm{Na}^{+}$ions could be built on an idea that removal of $\mathrm{K}^{+}$ions from their hydration shells is energetically more favorable than stripping hydration shells from $\mathrm{Na}^{+}$. This hypothesis could be potentially applied for MD and experimental studies. In addition, application of Donnan membrane principle to the ionic transport under CNT nanoconfinement could assist design and fabrication of devices for efficient separation, product recovery, synthesis of smart materials, and other innovative applications (Fornasiero, Park et al. 2008; Sarkar, SenGupta et al. 2010). These techniques use water as a process mediator and do not require either electricity or pressure gradient for sustained operation. It has potential of recovery of the alum in a high purity in a single-step process and reuse of it in the same plant or elsewhere as a coagulant. Also, the CNT based devises designed for ionic liquid transport and selectivity have potential applications in energy generation such as fuel cells, chemical nanoreactors, nanosensors and unipolar ionic field-effect transistors (Daiguji, Yang et al. 2004; Kang, Byun et al. 2004), since the high surface-to-volume ratio in CNT leads to ion separation via a transport mechanism governed by surface charges. While chapter 2 and 3 have discussed the progress and basic understandings of water and gas transport phenomena inside CNT, 
this chapter focuses on introductions of the MD simulation theories associated with ionic liquids inside CNT. In the first section, we will present an introduction to the basic theoretical models and formulas for MD simulations employed to describe the behavior of ionic liquids in nanochannels. Following sections will cover ion exclusion, selectivity and transport. Main aspects of the sections are summarized in the last section.

\subsection{MD simulation theories}

Today, molecular dynamics sees a widely spread use in the investigation of motion and interaction of biomolecules. Although MD simulation of real objects even at the nanometer scales is limited, success of MD in modeling liquids and gases under CNT nanoconfinement has been motivating researchers to utilize this tool in the analysis of the behavior of ionic solutions under CNT nanoconfinement. Fundamentally, MD combines Newtonian second law and interparticular potential energy.

$$
m_{i} \frac{\partial^{2} \vec{r}_{i}}{\partial t^{2}}=-\frac{\partial}{\partial \vec{r}_{i}} U_{T}\left(\vec{r}_{1}, \vec{r}_{2}, \ldots, \vec{r}_{N}\right), \quad i=1,2, \ldots, N,
$$

where $m_{i}$ is mass of a particle (atom or molecule) $i, r_{i}$ is its position, and $U_{T}$ is the potential energy that depends on atomic positions. Choice of the potential is the most critical as it can determine interactions between those particles in the simulation domain. To represent electrostatic interactions such as van der Waals attraction and Pauli repulsion, a mathematical function, viz. Lennard-Jones potential, is widely used. Contribution of the electrostatic charges of particles to the potential follows the Coulomb's law (Shao, Zhou et al. 2009).

$$
U\left(r_{i j}\right)=4 \varepsilon_{i j}\left[\left(\frac{\sigma_{i j}}{r_{i j}}\right)^{12}-\left(\frac{\sigma_{i j}}{r_{i j}}\right)^{6}\right]+\frac{q_{i} q_{j}}{r_{i j}},
$$

Equation (4.2) represents a typical interaction potential incorporating the electrostatic charge effect, in which $r_{i j}$ is the distance between particles $i$ and $j, q_{i}$ is the partial charge assigned to the particle $i$, and $\varepsilon_{i j}$ and $\sigma_{i j}$ are respectively energy and size parameters obtained by the Lorentz-Berthelot combining rule: potential vanishes at the interparticular distance of $\sigma_{i j}=\left(\sigma_{i}+\sigma_{j}\right) / 2$ and has a minimum well of $\varepsilon_{i j}=\left(\varepsilon_{i} \varepsilon_{j}\right)^{1 / 2}$. Poisson (Equation 4.3) and Nernst-Plank (Equation 4.4) equations are used for mathematical description of those potentials (Daiguji, Yang et al. 2004; Coalson and Kurnikova 2005; Peter and Hummer 2005).

$$
\begin{gathered}
\nabla^{2} \Phi(r)=-\frac{1}{\varepsilon_{0} \varepsilon} \sum_{i} z_{i} e n_{i}, \\
J_{i}=-D_{i}\left(\nabla n_{i}+\frac{z_{i} e n_{i}}{k_{\mathrm{B}} T} \nabla \phi\right),
\end{gathered}
$$

In Equations (4.3) and (4.4), $\varepsilon_{0}$ is the permittivity of vacuum, $\varepsilon$ is the solution dielectric constant, $n_{i}$ is the number density of ions of species $i, z_{i} e$ is their charge, $J_{i}$ is an ion flux, and 
$D_{i}$ is an ion diffusivity of species $i$. Equations (4.3) and (4.4) can calculate ionic current along a nanofluidic channel and surface charge density. When the tube surface is charged in a way that Debye length enlarges close to a pore radius, repellence of co-ions imparts ionic selectivity to the nanopore. In this manner, ion transport can be tuned by surface charge modulation (Daiguji, Yang et al. 2004). For ion transport and selectivity, concepts of energy penalty on CNT entry and water coordination shell number can play a major role. That is why analysis of ion hydration configuration in the bulk water and under CNT nanoconfinement is in scope of great interest. MD can determine the probabilities of the ion position $P(z, r)$ out of the radial distribution function. Free energy profile, $G(z)$, e.g. potential of mean force (PMF) for the CNT in one-dimensional case, could be calculated as follows:

$$
G(z)=-k_{\mathrm{B}} T \ln \int_{0}^{R} P(z, r) d r,
$$

where $R$ is the tube radius, $T$ is absolute temperature and $k_{\mathrm{B}}$ is Boltzmann's constant.

Some of ab initio MD (AIMD) take into account the polarizability of CNT in the framework of density functional theory (DFT) (Eschermann, Li et al. 2006; Leung and Marsman 2007; Aluru and $\mathrm{Xu}$ 2008). Polarizability of the tubes differs depending on delocalized $\Pi$-electrons. For example, an infinite polarizability of metallic CNT makes ion permeation less favorable than for the case of semiconducting CNT. The main action of large polarizability in ion permeation remains to be elucidated. Besides, huge computational cost prevents a wide spread use of the ab initio analysis in large structures, rendering it still remain a challenging task to calculate ion free energies compared with classical force fields approaches. Despite the difficulties, differences in polarizability could somehow be used for CNT characterization when proper experimental techniques would emerge. Aluru and $\mathrm{Xu}$ (Aluru and $\mathrm{Xu}$ 2008) showed that metallic SWNT has much stronger screening effects than semiconducting SWNT does when water molecules and ions permeates the nanotubes. These results imply that it would be possible to identify differently charged ions and to differentiate between metallic and semiconducting nanotubes. Leung and Marsman (Leung and Marsman 2007) carried out AIMD to demonstrate that intrinsic binding energies for metallic $(6,6)$ SWNT are 2.9 and $1.8 \mathrm{eV}$ for $\mathrm{Na}^{+}$and $\mathrm{Cl}^{-}$, respectively, while a much wider $(18,18)$ SWNT exhibit lower binding energies of 2.2 and $1.1 \mathrm{eV}$. This suggests that the proposed use of vertically aligned CNT in ion rejection membranes may need careful reexamination. They also pointed out that confined environment and molecular configurations can play a dominant role in the transport and selectivity processes. In this sense, ions behave in a similar manner in CNTs with diameters larger than $0.96 \mathrm{~nm}$, regardless of water configuration under nanoconfinements (Dzubiella and Hansen 2005; Peter and Hummer 2005; Corry 2007; Shao, Zhou et al. 2009; Beu 2010). The behavior of ions in larger diameter CNT agrees well with experimental results (Majumder, Chopra et al. 2005; Fornasiero, Park et al. 2008; Lee, Choi et al. 2010; Yu, Funke et al. 2010).

Unique properties not evident at macroscales can appear when liquids lie under nanoconfinement. In a narrow $\mathrm{CNT}$, the size of the tube could become commensurable with the size of the transporting molecules. In this situation, interaction between transporting molecules and CNT wall could emerge as a governing factor for the flow process, thereby nullifying continuum assumption and sometimes resulting in a single file configuration of 
water (Levinger 2002; Dong, Zhou et al. 2009). These unique nanoscale phenomena do not only apply to water molecular transport, but also to water as a processing chemical during ion transport and selectivity. The quantum state of the protons in the low temperature phase of water in nanotubes is qualitatively different from that of any phase of water seen so far. A transition temperature between bulk water and ice-like water phases is likely to depend on CNT size and details of the interaction between water molecules and the confining CNT. Should the phase exist at room temperatures for CNTs with different sizes, that would be of great interest in understanding the structure and transport of water in biological pores (Reiter, Burnham et al. 2006).

MD is a very powerful tool that helps us understand transport phenomena in CNT that are difficult or impossible to capture by state-of-the-art experimental techniques. Nevertheless, all models have their own restrictions and applied theories are mostly developed for macro scale. Hence, in applying these models directly to nanoscale, one should critically consider the validity of simulation results. Though, significance of molecular dynamics is arguably no less than experimental studies.

\subsection{Ion exclusion, selectivity and transport}

When it comes to the behavior of ionic liquids in CNT, it is difficult to draw a distinction among exclusion, selectivity and transport. Dissatisfaction of one process becomes gate conditions for the other. For example, cessation of ion transport means ion exclusion, and selectivity of ions implies exclusion of some of ion species and transport of the other species. Numerous simulations and experimental studies uncovered several possibilities in order to promote desirable processes. These possibilities range from the application of external pressure and electrical forces to the chemical addition of charged functional groups to the CNT opening or to the sidewall. Knowledge on the combination between driving force and chemical implantation per specific case would be crucial, as each combination may result in entirely different applications.

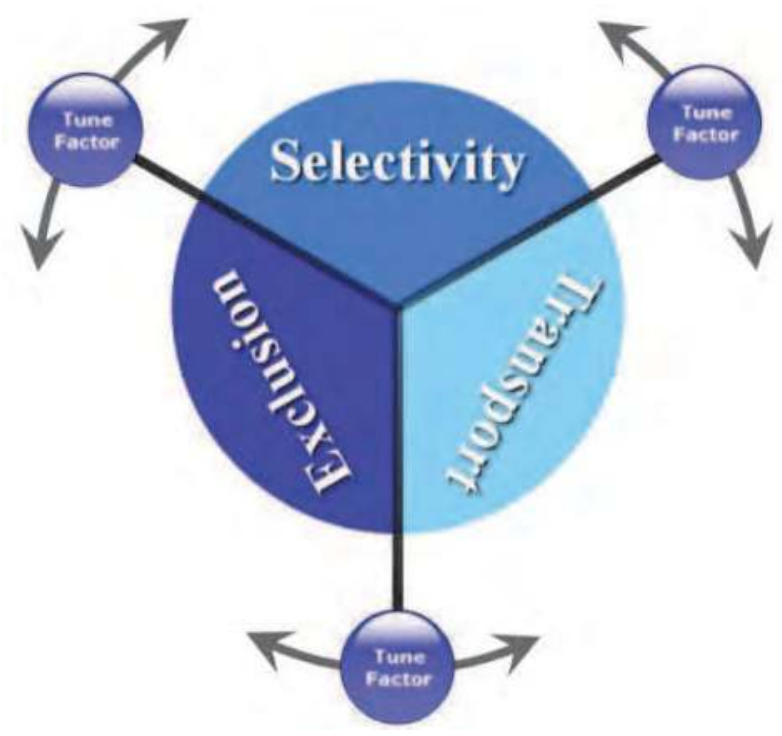




\subsection{1 lon exclusion}

Although water is the most abundant liquid in the planet, merely $3 \%$ is drinkable. Therefore, desalination and purification of seawater and brackish water are drawing a surge of attention today in association with future water scarcity. Fornasiero et al. (Fornasiero, Park et al. 2008) fabricated sub-2-nm CNT membranes and observed the ionic solution transport inside CNT. At the nanotube inlet, hydroxyl $(\mathrm{OH})$, carbonyl $(\mathrm{C}=\mathrm{O})$, and carboxylic $(\mathrm{COOH})$ functional groups are chemically added in order to impart a ring of negative charges to the CNT mouth. They examined ion transport and exclusion characteristics of this system, as a function of solution concentration, $\mathrm{pH}$, ion valence, and ion size. For the first time, they demonstrated that hydrophobic, sub-2-nm-wide CNT pores charged with negative moieties on the pore mouth can exclude ions and provide a strong indication that electrostatic interaction is responsible for ion rejections in CNT pores of this size (see Fig. 4.1). Their results made a good agreement with the Donnan membrane equilibrium theory, another evidence supporting the action of electrostatic interaction (Sarkar, SenGupta et al. 2010). Later, Banerjee et al. (Banerjee, Murad et al. 2007) pointed out that CNTs can be utilized for water and ion separation from a salt solution via three different electrostatic charge distributions on the pore surface (see Fig. 4.2). Molecular dynamics simulations showed that when these different charge distribution patterns are applied ion intake into the nanotubes is minimal, and negative charges on SWCNTs reduce the ion permeation to the tube that means it increase the value of the CNTs based devises as a water-purifying system. Goldsmith et al. (Goldsmith and Martens 2009) simulated an idealized model of membranes having surface-modified nanoporous of armchair CNTs of $(8,8),(10,10),(12,12),(14,14)$, and $(16,16)$. Nonequilibrium MD was applied for a pressurized flow of an aqueous $\mathrm{NaCl}$ solution for both uncharged CNT and CNT with artificial surface charge patterns (see Fig. 4.2). For uncharged CNT, the ionic flow rates showed a linear dependence on the driving pressure and increased with pore diameter. The amount of ion transport was orders of magnitude smaller than that of water, an evidence of salt rejection from the solution. Charged CNT, on the other hand, showed both water and ion transport rates smaller than those for uncharged CNT, and the smallest $(8,8)$ and $(10,10)$ systems exhibited nearly complete ion rejection. CNT surface modification with partial charges (Fig. 4.3) led to a decrease in overall flux of water but enhanced the amount of excluded salt from the system. Such enhancement of salt rejection could be achieved for larger pores.

As a membrane based distillation platform, Gethard et al. (Gethard, Sae-Khow et al. 2010) suggested a mixed matrix out of a polypropylene membrane embedded with uniformly distributed, PVDF surrounded MWNTs. It was the hydrophobic property of MWNT that augmented removal of the $\mathrm{NaCl}$ and $\mathrm{MgSO}_{4}$ salts from the ionic solution. Since water transport takes place in a vapor phase during the distillation process, water did not face any permeation barrier. In this way, permeated water purity was increased. One method of using a sheet network of chemically oxidized CNTs as absorbent of salts in ionic solutions was proposed by Tofighy et al. (Tofighy and Mohammadi 2010), with a decent degree of success. Subsequently, a capability of MWNT in adsorbing toxic heavy metal from water was experimentally described by Kandah et al. (Kandah and Meunier 2007) using oxidized MWNT ranging between 5.5 and $14 \mathrm{~nm}$. Oxidized CNT powders saw a certain degree of efficiency in adsorbing nickel from water solution. For example, negatively charged functional groups on CNT improved cation removal capacity. These oxidized CNT powders have excelled a commercial membrane (MINOTAUR) in the nickel removal capacity from water. However, we suppose that efficiency and efficacy of using CNT for water desalination and purification 
could be far more improved by use of CNT as membrane pore material than merely as absorbent or hydrophobic agent. The utilization of CNT as a basis channel for ion exclusion devices marks a promising route toward ion exclusion from aqueous solutions, attributed primarily to the capability of tailoring an entrance penalty of target ions and water. One of the best candidate mechanisms of ion exclusion is the manipulation of electrostatic interactions at the CNT pore, since the required ion exclusion could be achieved via rather simple methods such as functionalization of CNT or controlling $\mathrm{pH}$ of the ionic solution.

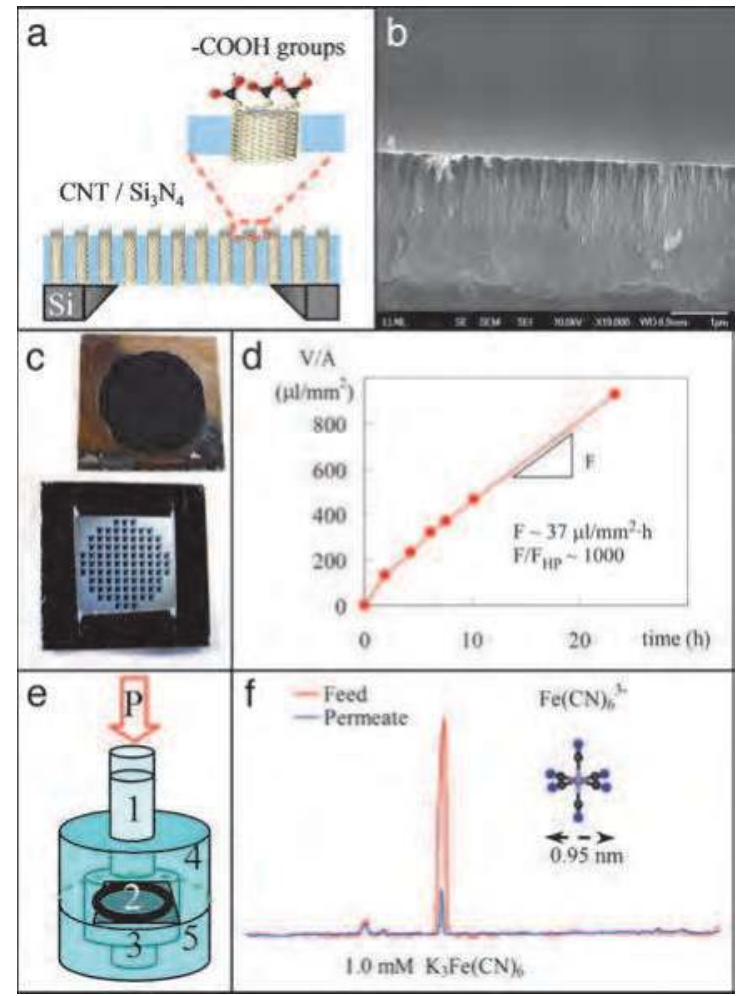

Fig. 4.1. CNT/silicon nitride membrane platform for ultrafast nanofiltration of electrolytes. (a) Cross-section schematic of a CNT membrane constituting a silicon support, aligned CNTs, silicon nitride matrix filling the gap between the CNTs, and the CNT tips functionalized with carboxylic groups. (b) Cross-section SEM image of the CNT/silicon nitride composite showing the gap-free coating of silicon nitride. (c) Photographs of the membrane sides exposed to the feed (upper) and to the permeate (lower). (d) Time variation of permeate volume per unit area of freestanding membrane during the filtration of a $0.6-\mathrm{mM} \mathrm{K}_{3} \mathrm{Fe}(\mathrm{CN})_{6}$ solution. The resulting permeation flux, F, is ca. 1,000 times larger than the value calculated by Hagen-Poiseuille equation, $\mathrm{F}_{\mathrm{HP}}$. (e) Schematic of the dead-end nanofiltration cell showing the column of feed solution (1) pressurized at $\mathrm{P}=0.69 \mathrm{bar}$, the CNT membrane (2), the permeate solution (3), and feed (4) and permeate (5) chambers. (f) Capillary electrophoresis chromatogram for feed (red) and permeate (blue) showing a 91\% exclusion of the ferricyanide anion after nanofiltration of a 1.0-mM K $\mathrm{K}_{3} \mathrm{Fe}(\mathrm{CN})_{6}$ solution (Fornasiero, Park et al. 2008). 


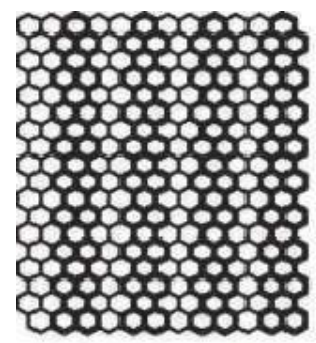

Circumferential ring

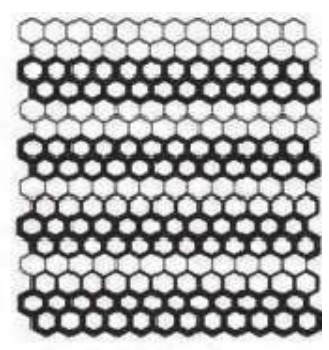

Axial band

Fig. 4.2. Schematic diagrams of the simulated charge distributions. The darker shade corresponds to a negative charge on a carbon atom while the lighter shade is for a positive charge (Banerjee, Murad et al. 2007).

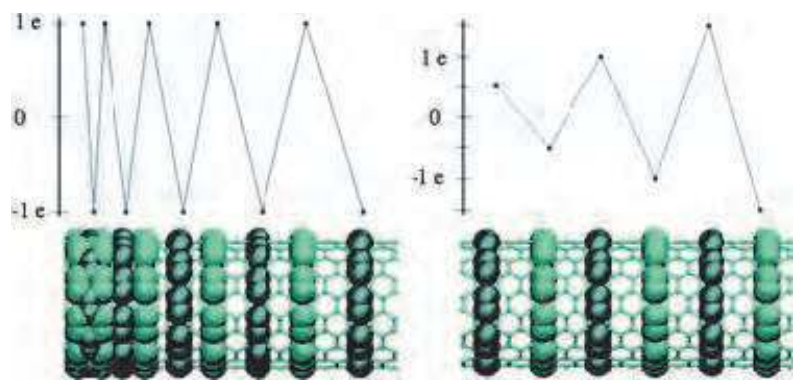

Fig. 4.3. Typical charge patterns employed for the surface-modified model CNTs. Partial charges are added to selected carbon atoms, as indicated in the figure by spheres of large radii in light and dark blue for positive and negative charges, respectively. The graph above each pore diagram indicates the total charge on the corresponding ring of carbon atoms. The left hand case consists of rings of equal amounts of charge, but an unequal spacing along the pore. The right hand case depicts equally spaced rings of charge, but increasing magnitudes along the pore (Goldsmith and Martens 2009).

\subsubsection{Ion selectivity}

Understanding and explanation of ion selectivity, for example in a potassium channel, are fundamental questions in nature, and therefore an inquiry of how channel can interact differently with $\mathrm{K}^{+}$and $\mathrm{Na}^{+}$ions have attracted much attention among researchers. Ions in aqueous solutions appear to find more energetically favorable when an ion is hydrated. When pore size is smaller than the dimension of the first hydration shell, the ion needs to get partially stripped of hydrating water molecules in order to gain entry to the pore, at the expense of energy penalty of the partial dehydration. This mechanism could be used for gated ionic transport via tailoring of the entrance energy barrier (Beckstein, Tai et al. 2004; Peter and Hummer 2005; Alexiadis and Kassinos 2008; Rasaiah, Garde et al. 2008).

Peter and Hummer (Peter and Hummer 2005) modeled a hexagonally packed CNT membrane to conclude that nonpolar pores with diameter around $5 \AA$ can block $\mathrm{Na}^{+}$ions completely, while they permeate the ions when the pore diameters increase to $10 \AA$. This finding was attributed to the interaction of hydration shell and pore in a way that an entropic loss equalizes 
to the potential barrier for ion penetration. Shao et al. (Shao, Zhou et al. 2009) investigated $\mathrm{Na}^{+}$ and $\mathrm{K}^{+}$selectivities inside CNTs with diameters $0.60,0.73,1.0,1.28$ and $2.00 \mathrm{~nm}$. Out of the five CNTs of their work, the first two small CNT were found favorable for confining a hydrated $\mathrm{K}^{+}$. On the contrary, the situation reversed in the wider CNTs with diameters of $0.87,1.0$, and $1.28 \mathrm{~nm}$. From this finding, they supposed that the ion selectivity is related to the energy cost of incorporating the hydrated cation under CNT confinement. Gong et al. (Gong, Li et al. 2010) also demonstrated how selectivity in $(9,9)$ SWNT could be improved by modification of the inner wall by eighth or sixteen carbonyl oxygen atoms. Their simulation showed a remarkable selectivity, attributed to the hydration structure of $\mathrm{Na}^{+}$or $\mathrm{K}^{+}$confined in the nanotube, which could further be precisely tuned by different patterns of the carbonyl groups. In the meantime, using MD simulation under equilibrium and nonequilibrium conditions, Dzubiella et al. investigated the ionic aqueous solution in a hydrophobic pore by focusing on dependence of ion and water permeations on pore diameter and electric field (Dzubiella and Hansen 2005). They reported that the energy barrier at the $\mathrm{CNT}$ pore entry was lower for the $\mathrm{K}^{+}$than for the $\mathrm{Na}^{+}$ions. Additionally, when the pore radius was smaller than a critical value, water had to be forced by an electric field to gain entry to the pore. The energy barrier that prevents ion penetration into CNT dropped to a few $k_{\mathrm{B}} T$ by increasing the electric field that polarized the channel. Similar results on ion selectivity in charged surroundings were also obtained by Yang et al. (Yang and Garde 2007). They studied the partitioning of cations of increasing size $\left(\mathrm{Na}^{+}\right.$, $\mathrm{K}^{+}$, and $\mathrm{Cs}^{+}$) in negatively charged $(5,5) \mathrm{CNT}$-like nanopore using MD. When the charge density of the pore was low, they found no entrance event of ions into CNT. An increase in the pore charge density, on the other hand, promoted the ion partitioning in the order $\mathrm{K}^{+}>\mathrm{Cs}^{+}>$ $\mathrm{Na}^{+}$, and the large $\mathrm{K}^{+}$and $\mathrm{Cs}^{+}$ions entered the CNT-like pore faster than small $\mathrm{Na}^{+}$ions. From this finding, they suggested an existence of a free energy barrier for the pore partitioning that could be adjusted via tailoring of pore charge density as shown in Table 4.1.

\begin{tabular}{cccccccccc}
\hline \hline & \multicolumn{3}{c}{ Nanopore (-2e) } & \multicolumn{3}{c}{ Nanopore (-5e) } & \multicolumn{3}{c}{ Nanopore (-8e) } \\
\cline { 2 - 11 } & $\Delta \mu_{I J}^{e x}$ & $\Delta \mu_{e l e}^{e x}$ & $\Delta \mu_{h y d}^{e x}$ & $\Delta \mu_{I J}^{e x}$ & $\Delta \mu_{e l e}^{e x}$ & $\Delta \mu_{h y d}^{e x}$ & $\Delta \mu_{I J}^{e x}$ & $\Delta \mu_{e l e}^{e x}$ & $\Delta \mu_{h y d}^{e x}$ \\
\hline $\mathrm{Na}^{+}$ & -7.0 & 68.8 & 61.8 & -7.1 & -92.6 & -99.7 & -6.9 & -276.8 & -283.7 \\
\hline $\mathbf{K}^{+}$ & 5.9 & 7.6 & 13.5 & 7.4 & -147.7 & -140.3 & 9.8 & -323.4 & -313.6 \\
\hline $\mathbf{C s}^{+}$ & 29.4 & -20.4 & 9.0 & 32.5 & -138.9 & -106.4 & 36.2 & -317.2 & -281.0 \\
\hline
\end{tabular}

Table 4.1. Free energies of water-to-hydrated-nanopore transfer of cations for three charge states of the nanopore. Contributions from LJ and electrostatic interactions are also listed. Units: kJ/mol (Yang and Garde 2007).

Later, Song et al. (Song and Corry 2009) demonstrated pressure controlled ion selectivity in narrow CNTs. For this purpose, they examined SWNTs with diameters of 3.4-6.1 $\AA$ to reveal that $\mathrm{Na}^{+}, \mathrm{K}^{+}$and $\mathrm{Cl}^{-}$ions face different energy barriers in entering the pore mouth of $\mathrm{CNT}$. For example, in the $(5,5)$ tube, the order of free energy barriers upon entry is $\Delta \mathrm{G}_{\mathrm{Cl}^{-}}>\Delta \mathrm{G}_{\mathrm{Na}}+$ $>\Delta \mathrm{G}_{\mathrm{K}+}$, which means that it is easiest for $\mathrm{K}^{+}$to enter the SWNT and the PMF barriers are no longer distinguishable. Major contribution to the free energy barrier difference is to bring about energy cost associated with a hydration shell of the ions. Ions in narrow pores could not gain a coordination number close to their bulk value. Selectivity in this case was governed by the ion solvation energy. Probably, this could also be more or less a correct 
description about the selectivity in potassium channels. Simulations and experimental studies have recognized the importance of a dimension of hydration shells around an ion in the ion selectivity. This implies a connection between the ions' behavior under CNT nanoconfinement and biological processes of human body and nature. Therefore, those studies could contribute to the discovery of the cure for ailments such as blindness, Alzheimer's disease, heart diseases and many others in the aging societies of the century.

\subsection{3 lonic liquid transport}

Ionic flow through the CNT can promote a lot of interests from industry because of energy applications as well as from the academia because of the fundamental impact of the basic transport mechanisms associated with it. Gated ion transport gives a perspective of using CNT as a storage container for the drug delivery systems, nanoreactors, molecular detectors, ionic transistors, rectifier circuits and many others. Insight obtained from ion transports under CNT nanoconfinement can be readily applied to the biological area via parallel CNT channels as a man-made analogy to biological ion channels that are able to generate coherent and oscillatory signals contingent upon physiological endo- and exocellular environments. By appling a simplified model of hydrophobic pores, Dzubiella et al. (Dzubiella and Hansen 2005) investigated cation transport in an aqueous solution under equilibrium and nonequilibrium conditions. Their simulation studies showed the intermittent filling of the hydrophobic cylindrical pore by water under equilibrium conditions, provided that the pore radius exceeds a critical value $R_{c}=5.3 \AA$. Below this critical radius, $R_{c}$, water could still permeate the pore under the action of a strong electric field. In addition, it was demonstrated that the hydrophobic pore has a lower entrance energy barrier for $\mathrm{K}^{+}$than that for $\mathrm{Na}^{+}$, despite the smaller size of the sodium ion. Recently, experiment and MD simulation implemented by Strano et al. (Strano, Kim et al. 2009) demonstrated a horizontal nanofluidic device. They used ionic solutions of $\mathrm{KCl}$, $\mathrm{NaCl}$, and $\mathrm{LiCl}$, induced the transport by a standard patch clamp technique, and traced currents for various applied electric fields $(600$ to $6000 \mathrm{~V} / \mathrm{m})$ across two reservoirs connected by up to 45 nanotubes that are $500 \mathrm{~mm}$ long as depicted in Fig. 4.4. They observed the stochastic pore blocking when individual cations partition into $\mathrm{CNT}$ obstructing an otherwise stable proton current. Domination of the proton conductance through the channel could be attributed to accumulation of protons and repulsion of anions from a negatively charged pore mouth etched by oxygen plasma. The measured proton transport is comparable with gramicidin proton channel, which recommends SWNT as an efficient candidate for a proton transport channel. Although stochastic signals in CNT look similar to those in biological channels, the transport and gate mechanisms of hydrated ions for CNT differ from those for biochannels, since the amount of negatively charged moieties CNT had at the pore mouth was not enough and ions had to be externally forced to enter the CNT. Their simulations assumed existence of optimal rate of proton diffusion through the nanopore that increases efficiency of ion transport from 5 ions/min to 480 ions/min. In other words, they observed single molecule ionic resonator. Ions can easily permeate $\mathrm{CNT}$ when external forcing is applied. According to MD simulation of electric field driven potassium ion transport in $(5,5)$ CNT (Kang, Byun et al. 2004), $\mathrm{K}^{+}$ions can enter CNT much faster when driven by external force field than driven by thermal fluctuations at equilibrium. This finding implies an application of data storage device via controllable gate ion transport in CNT. 


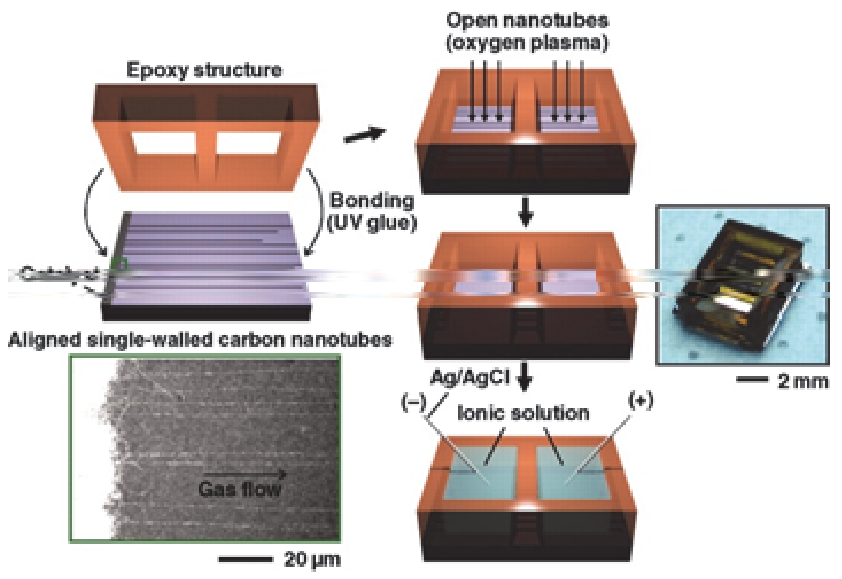

Fig. 4.4. Fabrication of SWNT ion channels. An epoxy structure with two compartments is bonded onto a substrate with ultralong and aligned CVD-grown nanotubes (scanning electron microscope image) (Strano, Kim et al. 2009).

Another MD simulation by Beu et al. (Beu 2010) investigated CNT diameter dependence of the ionic current. The simulations showed clearly that the ions in an aqueous solution inside the nanopore face significant energy barriers and can pass only through CNTs larger than $(7,7)$. The $(8,8)$ CNT does not appear to permeable ions very well, evidenced by rather low current measurement of about $0.05 \mathrm{nA}$ under all conditions. A six-fold increase in an ionic current was measured for the $(9,9)$ CNT with $0.68 \AA$ of channel. Qualitatively, the ion current or average number of ion passages through CNT exhibits a quadratic variation with respect to the CNT pore size and vanishes when the CNT diameter becomes about $1.1 \mathrm{~nm}$ corresponding to the $(8,8) \mathrm{CNT}$, as seen in Fig. 4.5.

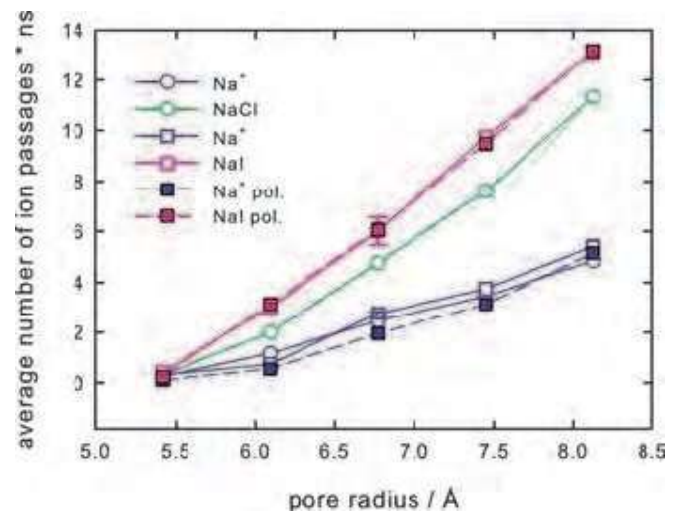

Fig. 4.5. Average total numbers of ion passages per nanosecond for the $\mathrm{NaCl}$ and $\mathrm{NaI}$ solutions as functions of the CNT radius. The partial currents due to the $\mathrm{Na}^{+}$ions only are plotted with blue lines and symbols. Filled symbols and dashed lines indicate the usage of polarizable models. The error bars represent the double difference between the values obtained using the time steps of 1 and $2.5 \mathrm{fs}$ (Beu 2010). 
Unlike MD simulations, experimental studies of ion transport through CNT do not abound. $\mathrm{Yu}$ et al. (Yu, Funke et al. 2010) experimentally investigated ion transport though a dense CNT membranes with both $3 \mathrm{~nm}$ CNT and $3 \mathrm{~nm}$ interstitial pores. They prepared this membrane with a solvent evaporation process, and used it as a model system for investigating an ion diffusion behavior in CNT. Interestingly, tuning of water wettability could observe a gated behavior of the ion diffusion through the CNT membrane. At the temperature of $298 \mathrm{~K}$, ion diffusion through these dense CNT membranes stopped after a few hours, but ion diffusion dramatically increased when the temperature elevated by $20 \mathrm{~K}$ and when the membrane was subjected to ultrasound sonication. Water adsorption isotherms of dense CNT membranes suggested that the adsorbed water may form a discontinuous phase at $293 \mathrm{~K}$, but it can quickly build a continuous layer at higher temperatures. When the temperature increase or ultrasound sonication opened up the ion diffusion channel, ions were found to diffuse through CNT at a rate similar to that of bulk diffusion in an aqueous solution. This finding may also bear implications for use of CNT membrane for desalination and water treatment. MD simulation of water assisted ionic transport in $(9,9)$ and $(10,10)$ CNTs performed by Dong et al. (Dong, Zhou et al. 2009) provided direct structural evidence about an anomalous phase behavior of the ionic liquid under CNT nanoconfinement. Although the entering numbers of cations and anions are equal when the CNT was saturated, cations always enter faster into the CNT channel than anions do during the entire filling process. The hydrogen bonding interaction plays a dominant role between cations and anions when an ionic liquid is confined by CNT. Consideration of free energy, which can determine a driving force of ions and ion pairs in entering CNT from its bulk phase, explained that a cation can enter CNT mostly by favorable dispersion of Lennard-Jones interactions. The free energy calculation revealed, however, that it is difficult for an anion to enter the CNT channel spontaneously. A more favorable case would be an ion pair entering CNT, with a cation "pulling" its counter anion by hydrogen bonding.

Water is required as a process mediator for the ion transport in CNT. One of the options to access gated ion transport in CNTs is to control the water flow inside the tube. Ionic current through CNT was found extremely dependent on CNT diameter. This clue offers one way of manipulating ion gating under CNT nanoconfinement. Another opportunity of controlling the ion flow in the nanochannels is to regulate electrostatic interactions between ions and $\mathrm{CNT}$ pore. It could be done via merge of concentration gradient, chemical addition of functional groups to CNT, polarization of $\mathrm{CNT}$, application of an external electric field and so on. Quite often, simulations based on highly simplified water potentials tend also to neglect the polarizability effect in the MD modeling. As of a length scale, a discrepancy exists between simulated CNT and CNT used in experimental verification, the former being ca. $1 \mathrm{~nm}$, while the latter being a few micrometers. All of these simplifications originated from a limit in computational cost could dramatically affect the validity of simulated data about ion selectivity and transport, unless care is taken.

\subsection{Summary}

Molecular dynamics is widely used to study the behavior of ions inside CNT, and yet it is not mature to capture the detailed essence of ionic configuration and transport under $\mathrm{CNT}$ nanoconfinement. Therefore, there exist emerging research opportunities. A variety of interatomic interaction potentials have been tested in order to describe water and ionic 
behaviors so far, however each potential has seen only a limited success in capturing the entire picture of those behaviors. In addition, computational cost sets a limit to the simulated CNT length scale of about one nanometer, which often forces researchers to ignore one important factor in modeling nanoconfined ions: polarizability. Therefore, the amount of research effort geared toward a description of ions' behavior under CNT nanoconfinement does not abound.

Exclusion, selectivity, and transport of ions are all much related processes. The close interplay between these concepts is based on the same basic mechanisms that govern ions behavior in CNT. In detail, in narrow CNT, it is the interaction between the water hydration shell of an ion and the narrow opening of CNT that plays a crucial role, primarily because of the commensurability in their dimensions. This interaction can especially become important to ion exclusion and selectivity. For CNTs with larger diameters, ion transport takes place more often than other phenomena. External forces applied to CNT can, however, provide a gated ion transport mechanism, imparting the selectivity component to the otherwise unselective ion transport. Functionalization of the CNT mouths and defect sites can strengthen an electrostatic interaction between ions and $\mathrm{CNT}$, giving another selectivity route to the ion transport. Incorporation of an electric current or control of a solution $\mathrm{pH}$ in the ion transport can also conjugate the selectivity mechanism. Combined control of different physical mechanisms in association with ions and CNT may enable practical selection among ion exclusion, ion selectivity, and ion transport.

Existing perspectives about the use of CNT as material for water purification and desalination are encouraging researchers to develop new models of simulation and experimental techniques and platforms for studying the ion exclusion phenomenon out of an aqueous solution. Analogy between CNT and biological channels brings a hope to the facilitated understanding of the mechanism of selectivity between potassium and sodium ions in human body. Ion transport under CNT nanoconfinement offers an opportunity for numerous applications such as a storage container for the drug delivery systems, nanoreactors, molecular detectors, ionic transistors and rectifier circuits. Finally, active interaction between simulation and experimental studies will allow us to better understand the fundamental science of ionic species under CNT nanoconfinement and to identify useful areas to apply the basic science to.

\section{Conclusion}

The recent development in nanotechnology enabled the integration of nanomaterials into conventional micro- and macrostructures, and thereby the probing of the novel nanoscale behaviour of mass transport or fluid dynamics of molecules confined in geometries commensurable with the molecular size.

In this chapter, we introduced and provided an overview of Carbon Nanotube Nanofluidics with three focal areas: water transport (section 2); gas transport (section 3); and ionic liquid transport (section 4). Carbon Nanotube Nanofluidics can be defined as both a discipline and a research area that pertains to mass transport under nanoscale confinement given by CNT. It studies nanoscale mass transport phenomena uniquely taking place inside CNT.

One common feature of these transports is the importance of molecular interaction under CNT nanoconfinement between transport entity and carbon wall. This interaction becomes dominant as the confinement tightens up. When water is placed in CNT, water molecules will experience a potential landscape provided by the hexagonal network of carbon atoms. It 
is hydrophobicity, atomic smoothness and curvature of CNT that are identified as the most important factors that determine water transport. As a result, water transport in large curvature CNTs exceeds any prediction of continuum formalism.

CNT can also transport gas efficiently. Transport mechanism of gas is characterized by a collision process. When gas enters CNT, its momentum transfer mechanism immediately shifts from gas-to-gas collision to gas-to-CNT collision. In this case, the atomic smoothness of CNT plays a primary role by augmenting specular collision between gas molecules and CNT wall, thereby enhancing the transport.

Ions inside CNT can take three pathways, i.e. exclusion, selectivity, and transport, depending on the dimensional comparison between CNT opening and effective hydration shell of ion. On top of the dimensional comparison, other physical mechanisms can also take part in selecting one of the ion transfer pathways. They include electrostatic interaction, electric field, and solution $\mathrm{pH}$.

Ultrafast transport of water and gas under $\mathrm{CNT}$ nanoconfinement can act as a major feature for energy efficient filtration, if combined with selectivity generation mechanisms. Noticeable avenues this concept could see a success encompass seawater desalination, water purification, demineralization, gas separation for greenhouse gas emission mitigation, rapid DNA sequencing, biomolecular separation, and so forth. Carbon Nanotube Nanofluidics is quickly combining fundamental nanoscience and socio-industrial needs, and poses potential for addressing future sustainability issues of humanity.

\section{References}

Agre, P. (2004). "Aquaporin water channels (Nobel lecture)." Angewandte ChemieInternational Edition 43(33): 4278-4290.

Agre, P. (2006). "Aquaporin water channels: from atomic structure to clinical medicine." Acta Pharmacologica Sinica 27: 27-27.

Agre, P., M. J. Borgnia, et al. (2001). "Discovery of the aquaporins and their impact on basic and clinical physiology." Aquaporins 51: 1-38.

Alexiadis, A. and S. Kassinos (2008). "Influence of water model and nanotube rigidity on the density of water in carbon nanotubes." Chemical Engineering Science 63(10): 27932797.

Alexiadis, A. and S. Kassinos (2008). "Molecular Simulation of Water in Carbon Nanotubes." Chemical Reviews 108(12): 5014-5034.

Aluru, N. R., C. Y. Won, et al. (2006). "Effect of quantum partial charges on the structure and dynamics of water in single-walled carbon nanotubes." Journal of Chemical Physics 125(11).

Aluru, N. R. and Y. Xu (2008). "Carbon nanotube screening effects on the water-ion channels." Applied Physics Letters 93(4).

ARKILIC, E. B., K. S. BREUER, et al. (2001). "Mass flow and tangential momentum accommodation in silicon micromachined channels." Journal of Fluid Mechanics 437: 29-43.

Arora, G. and S. I. Sandler (2006). "Air separation by single wall carbon nanotubes: Mass transport and kinetic selectivity." The Journal of Chemical Physics 124(8): 084702.

Banerjee, S., S. Murad, et al. (2007). "Preferential ion and water intake using charged carbon nanotubes." Chemical Physics Letters 434(4-6): 292-296.

Beckstein, O. (2003). "Liquid-vapor oscillations of water in hydrophobic nanopores." Proceedings of the National Academy of Sciences 100(12): 7063-7068. 
Beckstein, O., K. Tai, et al. (2004). "Not Ions Alone: Barriers to Ion Permeation in Nanopores and Channels." Journal of the American Chemical Society 126(45): 14694-14695.

Beskok, A. and G. E. Karniadakis (1999). "A model for flows in channels, pipes, and ducts at micro and nano scales." Microscale Thermophysical Engineering 3(1): 43-77.

Beskok, A., G. E. Karniadakis, et al. (1996). "Rarefaction and compressibility effects in gas microflows." Journal of Fluids Engineering-Transactions of the Asme 118(3): 448456.

Beu, T. A. (2010). "Molecular dynamics simulations of ion transport through carbon nanotubes. I. Influence of geometry, ion specificity, and many-body interactions." Journal of Chemical Physics 132(16).

Byl, O., J.-C. Liu, et al. (2006). "Unusual Hydrogen Bonding in Water-Filled Carbon Nanotubes." Journal of the American Chemical Society 128(37): 12090-12097.

Cannon, J. and O. Hess (2009). "Fundamental dynamics of flow through carbon nanotube membranes." Microfluidics and Nanofluidics 8(1): 21-31.

Chen, H. and D. S. Sholl (2006). "Prediction of selectivity and flux for CH4/H2 separations using single walled carbon nanotubes as membranes." Journal of Membrane Science 269(25): 152-160.

Coalson, R. D. and M. G. Kurnikova (2005). "Poisson-Nernst-Planck theory approach to the calculation of current through biological ion channels." Ieee Transactions on Nanobioscience 4(1): 81-93.

Cooper, S. M., B. A. Cruden, et al. (2003). "Gas Transport Characteristics through a Carbon Nanotubule." Nano Letters 4(2): 377-381.

Corry, B. (2007). "Designing Carbon Nanotube Membranes for Efficient Water Desalination." The Journal of Physical Chemistry B 112(5): 1427-1434.

Cui, H., O. Zhou, et al. (2000). "Deposition of aligned bamboo-like carbon nanotubes via microwave plasma enhanced chemical vapor deposition." Journal of Applied Physics 88(10): 6072-6074.

Daiguji, H., P. D. Yang, et al. (2004). "Ion transport in nanofluidic channels." Nano Letters 4(1): 137-142.

Daillant, J. (2009). "Recent developments and applications of grazing incidence scattering." Current Opinion in Colloid \& Interface Science 14(6): 396-401.

Dong, K., G. Zhou, et al. (2009). "Structural Evidence for the Ordered Crystallites of Ionic Liquid in Confined Carbon Nanotubes." The Journal of Physical Chemistry C 113(23): 10013-10020.

Dzubiella, J. and J. P. Hansen (2005). "Electric-field-controlled water and ion permeation of a hydrophobic nanopore." Journal of Chemical Physics 122(23).

Eschermann, J., Y. Li, et al. (2006). "Self-consistent ion transport simulation in carbon nanotube channels." Journal of Computational Electronics 5(4): 455-457.

Falk, K., F. Sedlmeier, et al. (2010). "Molecular Origin of Fast Water Transport in Carbon Nanotube Membranes: Superlubricity versus Curvature Dependent Friction." Nano Letters 10(10): 4067-4073.

Fedorov, A. S. and A. F. Sadreev (2009). "Ab-initio investigation of thermoactivated directional transport of hydrogen molecules inside narrow carbon nanotubes." physica status solidi (b) 246(11-12): 2598-2601.

Fornasiero, F., H. G. Park, et al. (2008). "Ion exclusion by sub-2-nm carbon nanotube pores." Proceedings of the National Academy of Sciences 105(45): 17250-17255.

Gethard, K., O. Sae-Khow, et al. (2010). "Water Desalination Using Carbon-NanotubeEnhanced Membrane Distillation." ACS Applied Materials \& Interfaces 3(2): 110114. 
Goldsmith, J. and C. C. Martens (2009). "Molecular Dynamics Simulation of Salt Rejection in Model Surface-Modified Nanopores." The Journal of Physical Chemistry Letters 1(2): 528-535.

Gong, X., J. Li, et al. (2010). "A Controllable Molecular Sieve for Na+ and K+ Ions." Journal of the American Chemical Society 132(6): 1873-1877.

Hanasaki, I., A. Nakamura, et al. (2008). "Structure and stability of water chain in a carbon nanotube." Journal of Physics: Condensed Matter 20(1): 015213.

Hanasaki, I. and A. Nakatani (2006). "Flow structure of water in carbon nanotubes: Poiseuille type or plug-like?" The Journal of Chemical Physics 124(14): 144708.

Hata, K., D. N. Futaba, et al. (2004). "Water-assisted highly efficient synthesis of impurityfree single-waited carbon nanotubes." Science 306(5700): 1362-1364.

Hinds, B. J., N. Chopra, et al. (2004). "Aligned multiwalled carbon nanotube membranes." Science 303(5654): 62-65.

Holt, J. K. (2006). "Fast Mass Transport Through Sub-2-Nanometer Carbon Nanotubes." Science 312(5776): 1034-1037.

Holt, J. K., A. Noy, et al. (2004). "Fabrication of a carbon nanotube-embedded silicon nitride membrane for studies of nanometer-scale mass transport." Nano Letters 4(11): 22452250.

Hummer, G., J. C. Rasaiah, et al. (2001). "Water conduction through the hydrophobic channel of a carbon nanotube." Nature 414(6860): 188-190.

Jakobtorweihen, S., C. P. Lowe, et al. (2007). "Diffusion of chain molecules and mixtures in carbon nanotubes: The effect of host lattice flexibility and theory of diffusion in the Knudsen regime." The Journal of Chemical Physics 127(2): 024904.

Joseph, S. and N. R. Aluru (2008). "Why Are Carbon Nanotubes Fast Transporters of Water?" Nano Letters 8(2): 452-458.

Kalra, A. (2003). "From The Cover: Osmotic water transport through carbon nanotube membranes." Proceedings of the National Academy of Sciences 100(18): 1017510180.

Kandah, M. I. and J.-L. Meunier (2007). "Removal of nickel ions from water by multi-walled carbon nanotubes." Journal of Hazardous Materials 146(1-2): 283-288.

Kang, J. W., K. R. Byun, et al. (2004). "Molecular dynamics study on the field effect ion transport in carbon nanotube." Physica E-Low-Dimensional Systems \& Nanostructures 24(3-4): 349-354.

Karniadakis, G., A. Beskok, et al. (2005). Microflows and Nanoflows: Fundamentals and Simulation (Interdisciplinary Applied Mathematics), Springer.

Kim, S., J. R. Jinschek, et al. (2007). "Scalable Fabrication of Carbon Nanotube/Polymer Nanocomposite Membranes for High Flux Gas Transport." Nano Letters 7(9): 28062811.

Koga, K., G. T. Gao, et al. (2001). "Formation of ordered ice nanotubes inside carbon nanotubes." Nature 412(6849): 802-805.

Kolesnikov, A. I., C. K. Loong, et al. (2006). "Anomalously soft dynamics of water in carbon nanotubes." Physica B: Condensed Matter 385-386(Part 1): 272-274.

Kowalczyk, P., L. Brualla, et al. (2007). "Single-Walled Carbon Nanotubes: Efficient Nanomaterials for Separation and On-Board Vehicle Storage of Hydrogen and Methane Mixture at Room Temperature?" The Journal of Physical Chemistry C 111(13): 5250-5257.

Kyakuno, H., K. Matsuda, et al. (2010). "Global Phase Diagram of Water Confined on the Nanometer Scale." Journal of the Physical Society of Japan 79(8): 083802. 
Lai, Z. P., G. Bonilla, et al. (2003). "Microstructural optimization of a zeolite membrane for organic vapor separation." Science 300(5618): 456-460.

Lee, C., C.-H. Choi, et al. (2008). "Structured Surfaces for a Giant Liquid Slip." Physical Review Letters 101(6): 064501.

Lee, C. Y., W. Choi, et al. (2010). "Coherence Resonance in a Single-Walled Carbon Nanotube Ion Channel." Science 329(5997): 1320-1324.

Lei, Y. and Y. Leng (2010). "Force oscillation and phase transition of simple fluids under confinement." Physical Review E 82(4).

Leung, K. and M. Marsman (2007). "Energies of ions in water and nanopores within density functional theory." Journal of Chemical Physics 127(15).

Levinger, N. E. (2002). "Water in Confinement." Science 298(5599): 1722-1723.

Li, J., C. Papadopoulos, et al. (1999). "Highly-ordered carbon nanotube arrays for electronics applications." Applied Physics Letters 75(3): 367-369.

Lin, Y., J. Shiomi, et al. (2009). "Dielectric relaxation of water inside a single-walled carbon nanotube." Physical Review B 80(4).

Loyalka, S. K. and S. A. Hamoodi (1990). "Poiseuille Flow of a Rarefied-Gas in a Cylindrical Tube - Solution of Linearized Boltzmann-Equation." Physics of Fluids a-Fluid Dynamics 2(11): 2061-2065.

Majumder, M., N. Chopra, et al. (2005). "Nanoscale hydrodynamics - Enhanced flow in carbon nanotubes." Nature 438(7064): 44-44.

Majumder, M., N. Chopra, et al. (2005). "Nanoscale hydrodynamics: Enhanced flow in carbon nanotubes (vol 438, pg 44, 2005)." Nature 438(7070): 930-930.

Majumder, M., N. Chopra, et al. (2005). "Effect of Tip Functionalization on Transport through Vertically Oriented Carbon Nanotube Membranes." Journal of the American Chemical Society 127(25): 9062-9070.

MajumderMainak, ChopraNitin, et al. (2005). "Erratum: Nanoscale hydrodynamics: Enhanced flow in carbon nanotubes." Nature 438(7070): 930-930.

Malek, K. and M.-O. Coppens (2003). "Knudsen self- and Fickian diffusion in rough nanoporous media." The Journal of Chemical Physics 119(5): 2801-2811.

Maruyama, S., E. Einarsson, et al. (2005). "Growth process of vertically aligned single-walled carbon nanotubes." Chemical Physics Letters 403(4-6): 320-323.

Mashl, R. J., S. Joseph, et al. (2003). "Anomalously Immobilized Water: A New Water Phase Induced by Confinement in Nanotubes." Nano Letters 3(5): 589-592.

Meyer, E. E. (2005). "Origin of the long-range attraction between surfactant-coated surfaces." Proceedings of the National Academy of Sciences 102(19): 6839-6842.

Mi, W., Y. Lin, et al. (2007). "Vertically aligned carbon nanotube membranes on macroporous alumina supports." Journal of Membrane Science 304(1-2): 1-7.

Miller, S. A., V. Y. Young, et al. (2001). "Electroosmotic Flow in Template-Prepared Carbon Nanotube Membranes." Journal of the American Chemical Society 123(49): 1233512342.

Miranda, L. D., R. T. Short, et al. (2009). "Direct coupling of a carbon nanotube membrane to a mass spectrometer: Contrasting nanotube and capillary tube introduction systems." Journal of Membrane Science 344(1-2): 26-31.

Naguib, N., H. H. Ye, et al. (2004). "Observation of water confined in nanometer channels of closed carbon nanotubes." Nano Letters 4(11): 2237-2243.

Newsome, D. A. and D. S. Sholl (2006). "Influences of Interfacial Resistances on Gas Transport through Carbon Nanotube Membranes." Nano Letters 6(9): 2150-2153.

Park, H. G. (2007). Mass Transport in Carbon Nanotubes. Mechanical Engineering. Berkeley, University of California Berkeley. Ph.D. 
Peter, C. and G. Hummer (2005). "Ion transport through membrane-spanning nanopores studied by molecular dynamics simulations and continuum electrostatics calculations." Biophysical Journal 89(4): 2222-2234.

Porodnov, B. T., P. E. Suetin, et al. (1974). "Experimental investigation of rarefied gas flow in different channels." Journal of Fluid Mechanics 64(03): 417-438.

Pozhar, L. A. (2000). "Structure and dynamics of nanofluids: Theory and simulations to calculate viscosity." Physical Review E 61(2): 1432.

Qiao, Y., G. Cao, et al. (2007). "Effects of Gas Molecules on Nanofluidic Behaviors." Journal of the American Chemical Society 129(8): 2355-2359.

Rasaiah, J. C., S. Garde, et al. (2008). "Water in Nonpolar Confinement: From Nanotubes to Proteins and Beyond *." Annual Review of Physical Chemistry 59(1): 713-740.

Raviv, U. (2002). "Fluidity of Bound Hydration Layers." Science 297(5586): 1540-1543.

Reiter, G., C. Burnham, et al. (2006). "Anomalous Behavior of Proton Zero Point Motion in Water Confined in Carbon Nanotubes." Physical Review Letters 97(24): 247801.

Roy, S., S. Cooper, et al. (2005). "Single component gas transport through 10nm pores: Experimental data and hydrodynamic prediction." Journal of Membrane Science 253(1-2): 209-215.

Sarkar, S., A. K. SenGupta, et al. (2010). "The Donnan Membrane Principle: Opportunities for Sustainable Engineered Processes and Materials." Environmental Science \& Technology 44(4): 1161-1166.

Sendner, C., D. Horinek, et al. (2009). "Interfacial Water at Hydrophobic and Hydrophilic Surfaces: Slip, Viscosity, and Diffusion." Langmuir 25(18): 10768-10781.

Shao, Q., J. Zhou, et al. (2009). "Anomalous Hydration Shell Order of $\mathrm{Na}+$ and $\mathrm{K}+$ inside Carbon Nanotubes." Nano Letters 9(3): 989-994.

Shiomi, J., T. Kimura, et al. (2007). "Molecular Dynamics of Ice-Nanotube Formation Inside Carbon Nanotubes." The Journal of Physical Chemistry C 111(33): 12188-12193.

Sholl, D. S. and J. K. Johnson (2006). "Making high-flux membranes with carbon nanotubes." Science 312(5776): 1003-1004.

Skoulidas, A., D. Ackerman, et al. (2002). "Rapid Transport of Gases in Carbon Nanotubes." Physical Review Letters 89(18).

Skoulidas, A. I., D. M. Ackerman, et al. (2002). "Rapid transport of gases in carbon nanotubes." Physical Review Letters 89(18).

Skoulidas, A. I., D. S. Sholl, et al. (2006). "Adsorption and diffusion of carbon dioxide and nitrogen through single-walled carbon nanotube membranes." Journal of Chemical Physics 124(5): -.

Sokhan, V. P., D. Nicholson, et al. (2001). "Fluid flow in nanopores: An examination of hydrodynamic boundary conditions." Journal of Chemical Physics 115(8): 38783887.

Sokhan, V. P., D. Nicholson, et al. (2002). "Fluid flow in nanopores: Accurate boundary conditions for carbon nanotubes." The Journal of Chemical Physics 117(18): 8531.

Sokhan, V. P., D. Nicholson, et al. (2004). "Transport properties of nitrogen in single walled carbon nanotubes." The Journal of Chemical Physics 120(8): 3855.

Song, C. and B. Corry (2009). "Intrinsic Ion Selectivity of Narrow Hydrophobic Pores." The Journal of Physical Chemistry B 113(21): 7642-7649.

Steckelmacher, W. (1986). "Knudsen flow 75 years on: the current state of the art for flow of rarefied gases in tubes and systems." Reports on Progress in Physics 49(10): 1083.

Steitz, R., T. Gutberlet, et al. (2003). "Nanobubbles and Their Precursor Layer at the Interface of Water Against a Hydrophobic Substrate." Langmuir 19(6): 2409-2418. 
Strano, M. S., W. J. Kim, et al. (2009). "Connecting Single Molecule Electrical Measurements to Ensemble Spectroscopic Properties for Quantification of Single-Walled Carbon Nanotube Separation." Journal of the American Chemical Society 131(9): 3128-+.

Suk, M. E., A. V. Raghunathan, et al. (2008). "Fast reverse osmosis using boron nitride and carbon nanotubes." Applied Physics Letters 92(13).

Takaiwa, D., K. Koga, et al. (2007). "Structures of filled ice nanotubes inside carbon nanotubes." Molecular Simulation 33(1): 127-132.

Thomas, J. A., R. M. Iutzi, et al. (2010). "Thermal conductivity and phonon transport in empty and water-filled carbon nanotubes." Physical Review B 81(4).

Thomas, J. A. and A. J. H. McGaughey (2008). "Reassessing Fast Water Transport Through Carbon Nanotubes." Nano Letters 8(9): 2788-2793.

Thornton, A. W., T. Hilder, et al. (2009). "Predicting gas diffusion regime within pores of different size, shape and composition." Journal of Membrane Science 336(1-2): 101108.

Tofighy, M. A. and T. Mohammadi (2010). "Salty water desalination using carbon nanotube sheets." Desalination 258(1-3): 182-186.

Verweij, H., M. C. Schillo, et al. (2007). "Fast Mass Transport Through Carbon Nanotube Membranes." Small 3(12): 1996-2004.

Waghe, A., J. C. Rasaiah, et al. (2002). "Filling and emptying kinetics of carbon nanotubes in water." The Journal of Chemical Physics 117(23): 10789.

Walther, J. H., R. Jaffe, et al. (2001). "Carbon Nanotubes in Water: Structural Characteristics and Energetics." The Journal of Physical Chemistry B 105(41): 9980-9987.

Wang, J., Y. Zhu, et al. (2004). "Diameter and helicity effects on static properties of water molecules confined in carbon nanotubes." Physical Chemistry Chemical Physics 6(4): 829-835.

Wang, Y., M. J. Kim, et al. (2005). "Continued Growth of Single-Walled Carbon Nanotubes." Nano Letters 5(6): 997-1002.

Wang, Y. Y., S. Gupta, et al. (2005). "Hollow to bamboolike internal structure transition observed in carbon nanotube films." Journal of Applied Physics 98(1).

Washburn, E. W. (1921). "The dynamics of capillary flow." Physical Review 17(3): 273-283.

Wei, B. Q., R. Vajtai, et al. (2002). "Microfabrication technology: Organized assembly of carbon nanotubes." Nature 416(6880): 495-496.

Whitby, M., L. Cagnon, et al. (2008). "Enhanced Fluid Flow through Nanoscale Carbon Pipes." Nano Letters 8(9): 2632-2637.

Willmott, G. R. and J. L. Tallon (2007). "Measurement of Newtonian fluid slip using a torsional ultrasonic oscillator." Physical Review E 76(6): 066306.

Wu, C. H., U. C. Sou, et al. (2006). "Influence of bicrystal microstructural defects on hightransition-temperature direct-current superconducting quantum interference device." Applied Physics Letters 88(10).

Yang, L. and S. Garde (2007). "Modeling the selective partitioning of cations into negatively charged nanopores in water." The Journal of Chemical Physics 126(8): 084706084708 .

Yu, M., H. H. Funke, et al. (2010). "Gated Ion Transport through Dense Carbon Nanotube Membranes." Journal of the American Chemical Society 132(24): 8285-8290.

Zhao, J., L. Liu, et al. (2009). "Thermal effect on the dynamic infiltration of water into singlewalled carbon nanotubes." Physical Review E 80(6).

Zhou, X.-Y. and H.-J. Lu (2007). "The structure and dynamics of water inside armchair carbon nanotube." Chinese Physics 16(2): 335. 


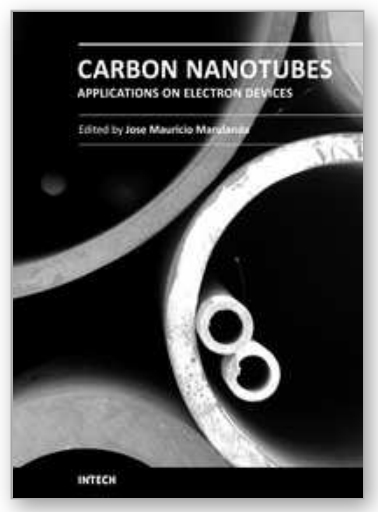

\section{Carbon Nanotubes Applications on Electron Devices}

Edited by Prof. Jose Mauricio Marulanda

ISBN 978-953-307-496-2

Hard cover, 556 pages

Publisher InTech

Published online 01, August, 2011

Published in print edition August, 2011

Carbon nanotubes (CNTs), discovered in 1991, have been a subject of intensive research for a wide range of applications. In the past decades, although carbon nanotubes have undergone massive research, considering the success of silicon, it has, nonetheless, been difficult to appreciate the potential influence of carbon nanotubes in current technology. The main objective of this book is therefore to give a wide variety of possible applications of carbon nanotubes in many industries related to electron device technology. This should allow the user to better appreciate the potential of these innovating nanometer sized materials. Readers of this book should have a good background on electron devices and semiconductor device physics as this book presents excellent results on possible device applications of carbon nanotubes. This book begins with an analysis on fabrication techniques, followed by a study on current models, and it presents a significant amount of work on different devices and applications available to current technology.

\section{How to reference}

In order to correctly reference this scholarly work, feel free to copy and paste the following:

Jong Won Choi, Maria Alexandrova and Hyung Gyu Park (2011). Carbon Nanotube Nanofluidics, Carbon Nanotubes Applications on Electron Devices, Prof. Jose Mauricio Marulanda (Ed.), ISBN: 978-953-307-496-2, InTech, Available from: http://www.intechopen.com/books/carbon-nanotubes-applications-on-electrondevices/carbon-nanotube-nanofluidics

\section{INTECH}

open science | open minds

\section{InTech Europe}

University Campus STeP Ri

Slavka Krautzeka 83/A

51000 Rijeka, Croatia

Phone: +385 (51) 770447

Fax: +385 (51) 686166

www.intechopen.com

\section{InTech China}

Unit 405, Office Block, Hotel Equatorial Shanghai

No.65, Yan An Road (West), Shanghai, 200040, China 中国上海市延安西路65号上海国际贵都大饭店办公楼 405 单元

Phone: +86-21-62489820

Fax: $+86-21-62489821$ 
(C) 2011 The Author(s). Licensee IntechOpen. This chapter is distributed under the terms of the Creative Commons Attribution-NonCommercialShareAlike-3.0 License, which permits use, distribution and reproduction for non-commercial purposes, provided the original is properly cited and derivative works building on this content are distributed under the same license. 